\title{
Molecular mechanism of reward treatment ameliorating chronic stress-induced depressive-like behavior assessed by sequencing miRNA and mRNA in medial prefrontal cortex
}

\author{
Tingting An \\ Qingdao University \\ Zhenhua Song \\ Qingdao University \\ Jin-Hui Wang ( $\nabla$ jhw@ibp.ac.cn ) \\ College of Life Science,University of Chinese Academy Sciences,Beijing China
}

\section{Research article}

Keywords: depression, reward, resilience, medial prefrontal cortex, miRNA

Posted Date: May 12th, 2020

DOl: https://doi.org/10.21203/rs.3.rs-23798/v1

License: (c) (1) This work is licensed under a Creative Commons Attribution 4.0 International License. Read Full License

Version of Record: A version of this preprint was published at Biochemical and Biophysical Research Communications on July 1 st, 2020 . See the published version at https://doi.org/10.1016/j.bbrc.2020.05.158. 


\section{Abstract}

Background Major depressive disorder (MDD) is a disease that seriously endangers human health and mental state. Chronic stress and lack of reward may reduce the function of the brain's reward circuits, leading to major depressive disorder. The effect of reward treatment on chronic stress-induced depression-like behaviors and its molecular mechanism in the brain remain unclear.

Methods Mice were divided into the groups of control, chronic unpredictable mild stress (CUMS), and CUMS-companion. Mice of CUMS group was performed by CUMS for 4 weeks, and CUMS-companion group was treated by CUMS accompanied with companion. The tests of sucrose preference, Y-maze, and forced swimming were conducted to assess depression-like behaviors or resilience. High-throughput sequencing was used to analyze mRNA and miRNA profiles in the medial prefrontal cortex harvested from control, CUMS-MDD (mice with depression-like behaviors in CUMS group), Reward-MDD (mice with depression-like behaviors in CUMS-companion group), CUMS-resilience (resilient mice in CUMS group), Reward-resilience (resilient mice in CUMS-companion group) mice.

Results The results provided evidence that accompanying with companion ameliorated CUMS-induced depression-like behaviors in mice. 45 differentially expressed genes (DEGs) are associated with depression-like behaviors, 8 DEGs are associated with resilience and 59 DEGs are associated with nature reward (companion) were identified. Furthermore, 196 differentially expressed miRNAs were found to be associated with companion. Based on the differentially expressed miRNAs and DEGs data, miRNA-mRNA network was established to be associated with companion.

Conclusion Taken together, our data here provided a method to ameliorate depression-like behaviors, and numerous potential drug targets for the prevention or treatment of depression.

\section{Background}

Major depressive disorder is clinically heterogeneous and common psychiatric disorder [1], and it is broadly characterized by persistent anhedonia, low self-esteem, markedly diminished interest or motivation accompanied by psychophysiological changes, such as sleep disturbances, changes in appetite or weight, lethargy or fatigue, psychomotor agitation or retardation, and impaired cognitive function [2-5]. The World Health Organization (WHO) reported that MDD will be the leading cause of disability and have the highest burden of disease by the year 2030 [6]. Studies on major depressive disorder have showed that lack of rewards in life can cause the brain's reward circuits to be not sufficiently activated or actively used, and may reduce the function of the brain's reward circuits $[7,8]$, including ventral tegmental area (VTA), nucleus accumbens (NAc) and prefrontal cortex (PFC), which may lead to major depressive disorder [9-11].

The reward circuit is part of the brain's limbic system, it not only promotes learning, stimulating and avoiding behavior, but also regulates people's cognition and motivation $[12,13]$. At the same time, recent studies have also demonstrated that disrupted topological organization within reward circuits was 
significantly associated with cognitive deficits and depression severity in MDD patients [13-15]. Generally, rewards can be divided into natural rewards and drug rewards. The former refers to innate desire or dependence on certain things, such as food, sounds, smells, food, sex, and social interactions $[16,17]$. The latter refers to the mental and physical dependence formed after long-term use of certain drugs or exposure to certain behaviors, also known as addiction $[18,19]$. Given the reward as a positive emotional stimulus [20], many studies have begun to investigate the effect of reward interventions on the improvement of depression symptoms. Studies have shown that music has an important role in emotional arousal and hedonic regulation [21-23] and music intervention can improve the symptoms of MDD, including sleep quality, quality of life, and lack of pleasure [24-26]. Similarly, studies have shown explicitly encouraging patients to participate in reward activities during treatment has been found to be effective in alleviating MDD [27]. And positive social feedback can alter emotional ratings and reward valuation of neutral faces [28]. Hence, increased attention to reward processing within the therapeutic process may improve clinical outcomes [29].

The reward circuit is heavily dependent upon ventral tegmental area (VTA) dopamine neurons and their targets, which include anterior cingulate cortex and medial prefrontal cortex (mPFC), cortex and nucleus accumbens (NAc) as well as additional corticostriatal circuitry involving the ventral pallidum, and diverse other structures spanning amygdala, hippocampus, thalamus, lateral habenula ( $\mathrm{LHb})$ and brainstem nuclei [20,30-32]. Studies demonstrates that in MDD patients, anhedonia is associated with weak communication between PFC and multiple brain regions important for reward, emotion and auditory processing [33-36]. Therefore, studying the molecular changes in the medial prefrontal cortex under reward treatment may provide better theoretical support for the application of reward intervention in the treatment of depression.

In this study, chronic unpredictable mild stress was used to produce depression-like behaviors mice or resilient mice, and accompanying with companion under the CUMS condition was performed as a reward intervention. The miRNA and mRNA profiles of mPFC was detected by high-throughput sequencing in depression-like behaviors mice or resilient mice derived from the two stress conditions. This research will explore the effect of reward treatment on CUMS-induced depression-like behavior, and further figure out the reward-related genes, which may be effective targets for the prevention and treatment of MDD.

\section{Methods}

Mice.

Three-week-old C57BL/6J mice were purchased from Beijing Vital River Laboratory Animal Technology Co., Ltd, and were maintained under controlled environmental conditions with free access to food and water, and illumination was provided between 07:00 and 19:00. The ambient temperature and relative humidity were maintained at $22 \pm 2{ }^{\circ} \mathrm{C}$ and $55 \pm 5 \%$, respectively. Experiments were performed in accordance with the guideline and regulation by Administration Office of Laboratory Animals at Beijing 
China, in which all Protocols were approved by Institutional Animal Care and Use Committee in this office (B10831).

Procedures for chronic unpredicted mild stress (CUMS) and accompanied by companion administration.

After the male mice at postnatal day 21 were purchased, they were acclimated for a week, in which body weight, sucrose preference test (SPT), Y-maze test (YMT) and forced swimming test (FST) were measured to have self-control data. SPT was used to assess anhedonia, YMT were used for the loss of interest to their partners, and FST was used for motivation-like behavior [37-41]. The SPT was tested by $1 \%$ sucrose water versus pure water in $4 \mathrm{~h}$, whose value was showed to be the ratio of the ingested sucrose water to the total amount of the ingested water (sucrose water plus pure water). The YMT was conducted by monitoring mice staying in a M-arm (the end of the arm was placed in a female mice) and other two arms for $2 \mathrm{~min}$. The value of YMT was showed by the ratio of stay time in M-arm to that in all three arms. The FST was performed by recording immobile time in a water cylinder $(10 \mathrm{~cm}$ in diameter and $19 \mathrm{~cm}$ in water depth at $25 \pm 1^{\circ} \mathrm{C}$ ). These tests were operated in a quiet room, no additional stresses, identical circadian circle for all mice and their adaptation in the test environment $[8,40]$.

According to the behavioral test data, mice of showing consistent values within mean $\pm 2 S D$ in these measurements were divided into three groups: control group (without CUMS), CUMS-group (treated by CUMS) and CUMS-companion group (treated by CUMS and accompanied with companion) (Fig. 1a). The CUMS procedure consisted of a variety of unpredictable mild stressors including empty cage, tilted cage, white noise, restraint space, damp sawdust cage, strobe light, social isolation and circadian disturbance, and were performed as previously described $[42,43]$. In the CUMS-companion group, mice were accompanied with female companion from the same litter for 30 min once per three days, when they were in the interval between two stressors (Fig. 1a). In this process, female mice and male mice are oneto-one corresponded, and after each end, these female mice were put back to the female cage.

Selecting and definition of MDD and resilience mice.

After CUMS or CUMS-companion treatments, behavioral tests were conducted to determine whether the mice developed anhedonia and lack of motivation. Mice with significant change in all three tests (values in the SPT and the YMT decreased above $20 \%$ of its self-control values as well as the immobile time of the FST increased $15 \%$ above its self-control values) were defined as CUMS-MDD mice in CUMS group, Reward-MDD mice in CUMS-companion group; Mice with $<5 \%$ change in all three tests were named as CUMS-resilience mice in CUMS group, Reward-resilience mice in CUMS-companion group. In addition, mice with significant changes in one or two tests but not all three tests were named as atypical mice. These standards are based on averaged values in our previous studies[8, 40,44].

RNA purification from the Medial Prefrontal cortex.

After CUMS-MDD, CUMS-resilience, Reward-MDD, Reward-resilience and control mice were selected, mice were euthanized. Firstly, mice were anesthetized with $0.1 \mathrm{ml} / 10 \mathrm{~g}$ intraperitoneal injection of $4 \%$ chloral 
hydrate. After the mice are fully anesthetized, perfused with $4{ }^{\circ} \mathrm{C}$ normal saline through the left atrium, and decapitated. The mPFC was separated on ice-cold glass slide. Total RNAs from mPFC were isolated in TRIzol Reagent (Life Technology, Carlsbad, CA, USA) as previously described [42], and RNA samples were delivered to Beijing Genomics Institute (BGI) in China for high-throughput sequencing analysis under dry ice conditions.

Library Preparation and mRNA-Sequencing.

mRNAs were purified with Oligo (dT)-attached magnetic beads, and sequentially fragmented into small pieces. Then, first-strand cDNA was synthesized using random hexamer-primed reverse transcription, followed by a second-strand cDNA synthesis. Obtained cDNA fragments were amplified by PCR, and products were purified by Ampure XP beads, then dissolved in EB solution. The product was validated on the Agilent Technologies 2100 bioanalyzer for quality control. Then, undergo DSN treatment. The DSN treated library was assessed quality to ensure the high quality of the sequencing data by two methods: checked the distribution of the fragments size using the Agilent 2100 bioanalyzer, and quantified the library using real-time quantitative PCR. The qualified library was amplified on cBot to generate the cluster on the flow cell. And the amplified flow cell was sequenced single end on the HiSeq4000 platform.

Differentially expressed transcript analysis and data analysis.

The reads per kilo-base per million reads (RPKM) was used to compute the level of transcript expression. Differentially expressed transcripts of samples were determined with DEseq.2. For screening differentially expressed transcripts, the threshold used to identify DEGs were |log2 fold change| $\geq 0.584936$ and FDR $\leq$ 0.05. Subsequently, DEGs were annotated in the gene ontology (G0) database (http://www.geneontology.org/) and Kyoto Encyclopedia of Genes and Genomes (KEGG) (http://www.genome.jp/kegg) database.

Library Preparation, miRNA-Sequencing and data analysis.

Library was prepared with $1 \mu \mathrm{g}$ total RNA for each sample. Total RNA was purified by electrophoretic separation on a 15\% urea denaturing polyacrylamide gel electrophoresis (GAGE) gel and small RNA regions corresponding to the 18-30 nt bands in the marker lane (14-30 ssRNA Ladder Marker, TAKARA) were excised and recovered. Then the18-30 nt small RNAs were ligated to a 5'-adaptor and a 3'-adaptor. The adapter-ligated small RNAs were subsequently transcribed into cDNA by SuperScript II Reverse Transcriptase (Invitrogen, USA) and then several rounds of PCR amplification with PCR Primer Cocktail and PCR Mix were performed to enrich the cDNA fragments. The PCR products were selected by agarose gel electrophoresis with target fragments 100-120 bp, and then purified by QIAquick Gel Extraction Kit (QIAGEN, Valencia, CA). The library was quality and quantitated in two methods: check the distribution of the fragments size using the Agilent 2100 bioanalyzer, and quantify the library using real-time quantitative PCR (TaqMan Probe). The final ligation PCR products were sequenced using the BGISEQ-500 platform. 
Subsequently, contaminated reads, including adapter dimers, junk, low complexity, common RNA families (rRNA, tRNA, snRNA, and snoRNA), were disregarded to obtain clean reads. Cleaned tags were annotated with miRBase 21.0 to identify known miRNAs. miRNA expression levels were calculated with TPM values [45]. DESeq software algorithm based on negative binomial distribution and biology duplicate samples was used to compare the known or novel miRNA expression among different groups. The threshold used to identify the different expression of miRNAs was fold-change larger than 1.5 and P-value $<0.05$. Three prediction approaches (RNAhybrid, Targetscan, and miRanda) were used to identify the miRNA binding sites.

Integrated miRNA/mRNA network analysis.

A series of bioinformatics analyses found that there is a correlationship between miRNAs and their target mRNAs. miRNAs were usually negatively correlated with their targeted mRNAs in theory, despite a few exceptions [46]. To identify potential miRNA-regulated target genes, the datasets of differentially expressed miRNAs and transcripts were integrated according to the following criteria: (1) In our analysis, miRNAs and mRNAs should undergo reverse changes simultaneously; (2) The correlationship between miRNAs and their target mRNAs should be predicted by the software of RNAhybrid, Targetscan or miRanda. Cytoscape software (San Diego, CA USA) was used to visualize the interactive network of differentially expressed miRNAs and concurrently expressed target mRNAs.

Quantitative RT-PCR for the validations of miRNA and mRNA.

Quantitative real-time RT-PCR (qRT-PCR) were performed with the Bio-Rad CFX 96Touch as previously described [47]. Briefly, total RNA was extracted from mPFC with a TRIzol Kit. cDNA was synthesized using the HiScript $\otimes$ RT SuperMix Reagent Kit (Vazyme, R323-01, China) for mRNA, and Mir-X miRNA FirstStrand Synthesis Kit (Clontech, 638315, CA, USA) for miRNA. mRNAs were amplified in a $20 \mu$ l reaction with $1 \mu \mathrm{l}$ sample cDNA (500 ng/ $\mu \mathrm{l}), 0.4 \mu \mathrm{l}$ of each primer $(10 \mathrm{nmol} / \mathrm{l}), 10 \mu \mathrm{l} 2 \times$ ChamQ Universal SYBR qPCR Master Mix and $8.2 \mu \mathrm{lddH_{2 }}$ O. Real-time PCR was initiated at $95^{\circ} \mathrm{C}$ for $30 \mathrm{~s}$, followed by 40 cycles of denaturation for $10 \mathrm{~s}$ at $95^{\circ} \mathrm{C}$, annealing and elongation for $30 \mathrm{~s}$ at $60^{\circ} \mathrm{C}$, then melt curve $95^{\circ} \mathrm{C}$ for $15 \mathrm{~s}, 60^{\circ} \mathrm{C}$ for $1 \mathrm{~min}$, and $95^{\circ} \mathrm{C}$ for $15 \mathrm{~s}$. For miRNAs, qRT-PCR was performed in a $20 \mu$ reaction with $1.6 \mu \mathrm{l}$ sample cDNA, $0.4 \mu \mathrm{l}$ mRQ3'Primer, $0.4 \mu \mathrm{l}$ miRNA-specific Prime $(10 \mu \mathrm{M}), 10 \mu \mathrm{l} 2 \times$ SYBR Advantage Premix, and $7.6 \mu \mathrm{lddH_{2 }}$ O. The program was set to $95^{\circ} \mathrm{C}$ for $10 \mathrm{~s}$, followed by 40 cycles of denaturation for $5 \mathrm{~s}$ at $95^{\circ} \mathrm{C}$, annealing and elongation for $20 \mathrm{~s}$ at $60^{\circ} \mathrm{C}$ and melt curve $65^{\circ} \mathrm{C}$ to $95^{\circ} \mathrm{C}$ increment $0.5^{\circ} \mathrm{C}$ for $5 \mathrm{~s}$. The relative expression level of mRNAs in the tissue was normalized to an internal reference gene GAPDH. The relative expression level of miRNAs in the tissue was normalized to U6 small nucleolar RNA. Each amplification was performed in triplicate. The results were calculated with the $2^{-\Delta \Delta C t}$ method.

Dual luciferase reporter assay.

According to the miRNA/mRNA prediction, targeted gene sequence containing targeted sites was amplified by PCR. Then, the products were digested by Xhol and Notl and fused into the luciferase vector psiCHEK2 [44]. Site-directed mutation of targeted site was performed with QuikChange Lighting Site- 
Directed Mutagenesis Kit (Stratagene, La Jolla, CA) according to the manufacturer's instructions. Luciferase reporter detection assays were carried out as previously described [48]. Briefly, $50 \mathrm{ng}$ psiCHECK2-wild-type or mutant reporter plasmids, $50 \mathrm{nM}$ miRNAs mimic or miR-NC mimic were cotransfected to HEK293T cells by using Lipofectamine 2000 transfection reagent (Invitrogen, Carlsbad, CA, USA). After $24 \mathrm{~h}$, the activities of firefly and Renilla luciferase were assessed by using Dual-Glo® Luciferase Assay System (Promega, Cat. E2920, USA) according to the manufacturer's instructions. Each treatment was carried out in triplicates in three independent experiments.

Statistical analyses.

The data of behavioral tests, luciferase activity and gene analyses are presented as mean \pm SEM. Relationships between miRNA and its target prediction were assessed by Pearson's correlation coefficients. Two-way ANOVA was used to make the statistic comparison among control, CUMS and CUMS-companion groups before and after treatment. Paired t-test was used for statistics of before versus after values within groups. The unpaired Student $t$-test was used to make the statistic comparison between control and CUMS-MDD, control and CUMS-resilience, control and Reward-MDD, control and Reward-resilience, CUMS-resilience and Reward-resilience, and so on. $\mathrm{P}<0.05$ is considered statistically significant.

\section{Results}

Accompanying with companion ameliorated CUMS-induced depression-like behaviors in mice.

In order to identify the effect of rewards on ameliorating depression-like behaviors, as a classic depression model [49], CUMS model was used to explore the possible antidepressant effect of accompanying with companion. As shown in Fig. 1a, C57BL/6J male mice were treated with CUMS or CUMS-companion for 4 weeks, after which depression-like behaviors or resilience were assessed by sucrose preference test, Y-maze test and forced swimming test. According to the behavioral tests, only mice changed significantly in all three tests could they be defined as MDD mice, and mice did not change significantly in all three tests could they be defined as resilience mice (Fig. 1a). The results showed that three behavioral parameters of the CUMS and CUMS-companion groups varied so much after 4 weeks treatments (Fig. 1b-d). In CUMS group mice, the SPT values significantly decreased after 4 weeks treatments ( $83.86 \pm 0.9225 \%$ versus $71.51 \pm 1.788 \%, p<0.0001), n=67$ )(Figure $1 b$ and Table $S 1$ ), the ratios of stay time in $\mathrm{M}$-arm to stay time in total arms also showed significant difference after treatments (78.54 $\pm 1.177 \%$ versus $57.22 \pm 2.05 \%, p<0.0001, n=67$ ) (Fig. $1 \mathrm{c}$ and Table S2), and the FST's immobile time were $206.6 \pm 7.086$ seconds after treatments and $137 \pm 6.935$ seconds before the CUMS treatments $(p<0.0001, n=67)$ (Fig. 1d and Table S3). These results indicated that CUMS treatments can induce depression-like behaviors in mice.

It is noteworthy that pairwise comparisons between the three groups after CUMS showed significant differences in SPT, YMT and FST, respectively (Fig. 1b-d, Table S1-3). Compared with CUMS group, the SPT and YMT values significantly increased in CUMS-companion group, and the ratios of stay time in M- 
arm to stay time in total arms showed significantly decrease in CUMS-companion group (Fig. 1b-d, Table S1-3). In addition, as indicated in Table 1, mice treated by the CUMS or CUMS and companion in 4 weeks, the percentage of MDD in CUMS group was about 29.85\%, while that in CUMS-companion group decreased to $12.12 \%$. Meanwhile, the percentage of resilience in CUMS group was about $11.94 \%$, while that in CUMS-companion group increased to $36.36 \%$. These results suggested that accompanying with companion ameliorated CUMS-induced depression-like behaviors in mice.

\section{Table 1}

Behavioral changes in the group of CUMS and CUMS-companion mice.

\begin{tabular}{|lllll|}
\hline Mice & CUMS & Percentage (\%) & CUMS-companion & Percentages (\%) \\
\hline MDD & 20 & 29.85 & 8 & 12.12 \\
\hline Resilience & 8 & 11.94 & 24 & 36.36 \\
\hline Atypical & 39 & 58.21 & 34 & 51.52 \\
\hline Total & 67 & 100 & 66 & 100 \\
\hline
\end{tabular}

Accompanying with companion disturbed the mRNA expression of medial prefrontal cortex in CUMSinduced MDD and resilience mice.

To investigate the molecular mechanism of ameliorated effect of accompanying with companion in CUMS-induced depression-like behaviors, the miRNA and mRNA profiles were analyzed by highthroughput sequencing in medial prefrontal harvested from control, CUMS-MDD, Reward-MDD, CUMSresilience, Reward-resilience mice. 15 samples were sequenced using RNA-Seq technology, and the number of raw sequencing reads and clean reads are shown in Supplementary Table S4. The unique mapping ratio with reference gene and the average genome mapping ratio were more than $79.48 \%$ (Supplementary Table S4). The criterion to make sure differential expression of genes were a 1.5-fold or greater change in transcript level between any two group mice and a P-value $<0.05$. As shown in Supplementary Table S5, 311 differentially expressed mRNAs were obtained in control versus CUMSMDD mice, in which 195 mRNAs were up-regulated and 116 mRNAs were down-regulated in CUMS-MDD mice compared to control mice. In the control versus Reward-MDD mice, 127 differentially expressed mRNAs changed in Reward-MDD mice compared to control mice, where 21 mRNAs were down-regulated and 106 mRNAs were up-regulated. For resilience mice derived from two treatments, 45 differentially expressed mRNAs were obtained in control versus CUMS-resilience mice, in which 24 mRNAs are upregulated and 21 mRNAs are downregulated; 45 differentially expressed mRNAs were obtained in control versus Reward-resilience mice, in which 22 mRNAs are upregulated and 23 mRNAs are downregulated. These results indicate that the molecular changes in the medial prefrontal region are different in different treatments that achieve consistent behaviors.

In order to validate the sequencing data above, we ran quantitative RT-PCR (qRT-PCR) from tissues that were used for mRNA sequencing. As shown in Supplementary Figure S1-S3, the expressions of Cc/28, Ciart, Fkbp5, Fmo2, Gm7120, Gpr149, Hif3a, Kcnh5, Npas4, Plin4, Xdh were decreased, as well as the 
expressions of Slc6a13 was increased in Reward-MDD mice, compared to control mice (Figure S1). Moreover, the expressions of Ciart, Dbp, Fmo2, Hif3a, Lrrc39, Xdh were decreased, as well as the expressions of Col3a1, Pcdha1, S/c6a13 and Stac were increased in Reward-resilience mice, compared to control mice (Figure S2). Consistent results achieved by mRNA sequencing and qRT-PCR confirm the validation of our study.

Identification of differentially expressed genes are associated with depression-like behaviors.

Since the depression-like behaviors in MDD mice were consistent whether they were derived from CUMS treated group or CUMS-companion group, the common differentially expressed genes (DEGs) in control versus CUMS-MDD and control versus Reward-MDD comparisons may be strongly associated with depression-like behaviors. To identify both unique and common genes in control versus CUMS-MDD and control versus Reward-MDD comparisons, numbers were calculated and presented using a Venn diagram (Fig. 2). As shown in Fig. 2 and Supplementary Table S6, 266 DEGs (I) specifically involved in control versus CUMS-MDD comparison, and 82 unique DEGs (III) in control versus Reward-MDD comparison. In addition, we obtained 45 common DEGs (II) in control versus CUMS-MDD and control versus RewardMDD comparisons, such as AnIn, Ano2, Atp10a, Ccdc152, Ccdc187, Ccl28, Cd44, Cdh19, Clic5, Cpm, Crb1, Ecm1, Etnppl, Fmo2, Fmod, Gm14440, Gm15446, Gm21949, Gm7120, Gpt, Grin1os, Hapln2, Hhip, Hif3a, Htr4, II12rb1, Kdr, Mertk, Nptx2, Pcdha4, Plin4, Rcn3, Sdc4, Sec14I5, Slc6a13, Slco4a1, Smad6, Smim3, St18, Tmem88, Tsc22d3, Ugt8a, Wdr86, Xdh and 1700047M11Rik. More importantly, the expression pattern of each common gene was consistent in both CUMS-MDD and Reward-MDD mice compared to control mice, either the expression simultaneously increased or decreased.

To gain in-depth insights into the molecular function of these unique and common DEGs, we performed Kyoto Encyclopedia of Genes and Genomes pathway enrichment analysis. As shown in Fig. 2 and Supplementary Table S6, 266 unique DEGs (I) were found to be mainly enriched in KEGG pathways associated with Metabolic pathways, PI3K-Akt signaling pathway, Wnt signaling pathway, Hippo signaling pathway, Cytokine-cytokine receptor interaction, ECM-receptor interaction, Neuroactive ligandreceptor interaction, MAPK signaling pathway, Axon guidance and Inflammatory mediator regulation of TRP channels. 82 unique DEGs (III) were found to be mainly enriched in KEGG pathways associated with Metabolic pathways, MAPK signaling pathway, ECM-receptor interaction, Serotonergic synapse, Dopaminergic synapse and Neuroactive ligand-receptor interaction. What is more, 45 common DEGs (II) were mainly enriched in KEGG pathways associated with Metabolic pathways, CAMP signaling pathway, ECM-receptor interaction, PI3K-Akt signaling pathway, Serotonergic synapse and Neuroactive ligandreceptor interaction.

Identification of differentially expressed genes are associated with resilience.

Similar to the depression-like behaviors of MDD mice, the behaviors of resilience mice derived from CUMS treated group and CUMS-companion group were also consistent. Therefore, the common differentially expressed genes (DEGs) are associated with resilience were identified, calculated and presented using a Venn diagram (Fig. 3). As shown in Fig. 3 and Supplementary Table S7, 37 DEGs (I) 
specifically involved in control versus CUMS-resilience comparison, and 37 unique DEGs (III) in control versus Reward-resilience comparison. In addition, we obtained 8 common DEGs (II) in control versus CUMS-resilience and control versus Reward-resilience comparisons, such as Cpm, Fmo2, Grin1os, Hif3a, Hist2h2aa2, Plin4, Scn5a and Trex1. What is more, the expression pattern of each common gene was consistent in both CUMS-resilience and Reward-resilience mice compared to control mice, either the expression simultaneously increased or decreased. Compared with MDD mice, the resilience mice derived from CUMS treated group and CUMS-companion group seem to share less molecular changes.

Subsequently, Kyoto Encyclopedia of Genes and Genomes pathway enrichment analysis was performed for unique DEGs (I), unique DEGs (III) and common DEGs (II). As shown in Fig. 3 and Supplementary Table S7, 37 unique DEGs (I) were found to be mainly enriched in KEGG pathways associated with cAMP signaling pathway, Neuroactive ligand-receptor interaction, Metabolic pathways, PI3K-Akt signaling pathway, Wnt signaling pathway, ECM-receptor interaction, Regulation of actin cytoskeleton and Tight junction. 37 unique DEGs (III) were found to be mainly enriched in KEGG pathways associated with ECMreceptor interaction, PI3K-Akt signaling pathway, Metabolic pathways, p53 signaling pathway, Alcoholism, Caffeine metabolism, Biosynthesis of amino acids and Rap1 signaling pathway. What is more, 8 common DEGs (II) were mainly enriched in KEGG pathways associated with Alcoholism and Adrenergic signaling in cardiomyocytes.

Identification of differentially expressed genes are associated with accompanying with companion.

Most individuals may not suffer from major depression by chronic stress, i.e., resilience [50]. As shown in Table 1, approximately $11.94 \%$ of CUMS-treated mice in 4 weeks were resilience, and this value increased to approximately $36.36 \%$ after accompanying with companion. To identify the DEGs are associated with the accompanying with companion treatments, differentially expressed mRNAs were screened in CUMSresilience versus Reward-resilience mice. As shown in Supplementary Table S5, 59 differentially expressed mRNAs were obtained in CUMS-resilience versus Reward-resilience mice, in which 26 mRNAs were up-regulated and 33 mRNAs were down-regulated in CUMS-MDD mice compared to control mice. To gain in-depth insights into the molecular function of these differentially expressed mRNAs, we performed KEGG pathway enrichment analysis. As shown in Table 2, differentially expressed mRNAs were found to be mainly enriched in KEGG pathways associated with Neuroactive ligand-receptor interaction, Dopaminergic synapse, Rap1 signaling pathway, Focal adhesion, Metabolic pathways and PI3K-Akt signaling pathway. 
Table 2

Signaling pathways identified by KEGG function analysis based on DEGs data in CUMS-resilience versus Reward-resilience.

\begin{tabular}{|c|c|c|c|}
\hline KEGG Entry & Term & Count & Genes \\
\hline \multirow[t]{2}{*}{ mmu04510 } & Focal adhesion & 3 & $\begin{array}{l}\text { Rac3 (RAS-related C3 botulinum substrate 3) } \downarrow \text {, } \\
\text { Col6a3 (collagen, type VI, alpha 3) } \downarrow \text {, }\end{array}$ \\
\hline & & & Thbs1 (thrombospondin 1) $\uparrow$ \\
\hline mmu05219 & Bladder cancer & 2 & $\begin{array}{l}\text { Mmp9 (matrix metallopeptidase 9) } \downarrow \text {, Thbs } 1 \\
\text { (thrombospondin 1) } \uparrow\end{array}$ \\
\hline \multirow[t]{2}{*}{ mmu04015 } & $\begin{array}{l}\text { Rap1 signaling } \\
\text { pathway }\end{array}$ & 3 & $\begin{array}{l}\text { Rac3 (RAS-related C3 botulinum substrate 3) } \downarrow \text {, Drd2 } \\
\text { (dopamine receptor D2) } \downarrow \text {, }\end{array}$ \\
\hline & & & Thbs1 (thrombospondin 1) $\uparrow$ \\
\hline mmu04115 & $\begin{array}{l}\text { p53 signaling } \\
\text { pathway }\end{array}$ & 2 & 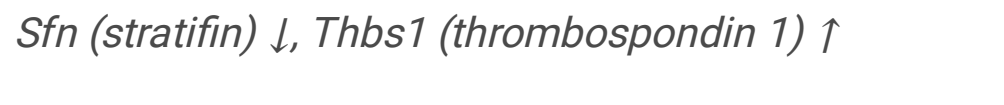 \\
\hline mmu04512 & $\begin{array}{l}\text { ECM-receptor } \\
\text { interaction }\end{array}$ & 2 & $\begin{array}{l}\text { Col6a3 (collagen, type VI, alpha 3) } \downarrow \text {, Thbs } 1 \\
\text { (thrombospondin 1) } \uparrow\end{array}$ \\
\hline mmu04974 & $\begin{array}{l}\text { Protein digestion } \\
\text { and absorption }\end{array}$ & 2 & $\begin{array}{l}\text { Col9a2 (collagen, type IX, alpha 2) †, Col6a3 } \\
\text { (collagen, type VI, alpha 3) } \downarrow\end{array}$ \\
\hline $\mathrm{mmu04728}$ & $\begin{array}{l}\text { Dopaminergic } \\
\text { synapse }\end{array}$ & 2 & $\begin{array}{l}\text { Drd2 (dopamine receptor } D 2) \downarrow \text {, Drd4 (dopamine } \\
\text { receptor } D 4) \downarrow\end{array}$ \\
\hline mmu04550 & $\begin{array}{l}\text { Signaling pathways } \\
\text { regulating } \\
\text { pluripotency of } \\
\text { stem cells }\end{array}$ & 2 & $\begin{array}{l}\text { Lhx5 (LIM homeobox protein 5) †, KIf4 (Kruppel-like } \\
\text { factor 4) } \uparrow\end{array}$ \\
\hline mmu04024 & $\begin{array}{l}\text { CAMP signaling } \\
\text { pathway }\end{array}$ & 2 & $\begin{array}{l}\text { Rac3 (RAS-related C3 botulinum substrate } 3 \text { ) } \downarrow \text {, Drd2 } \\
\text { (dopamine receptor D2) } \downarrow\end{array}$ \\
\hline mmu05205 & $\begin{array}{l}\text { Proteoglycans in } \\
\text { cancer }\end{array}$ & 2 & $\begin{array}{l}\text { Mmp9 (matrix metallopeptidase 9) } \downarrow, \text { Thbs } 1 \\
\text { (thrombospondin 1) } \uparrow\end{array}$ \\
\hline mmu05206 & $\begin{array}{l}\text { MicroRNAs in } \\
\text { cancer }\end{array}$ & 2 & $\begin{array}{l}\text { Mmp9 (matrix metallopeptidase 9) } \downarrow \text {, Thbs } 1 \\
\text { (thrombospondin 1) } \uparrow\end{array}$ \\
\hline mmu04080 & $\begin{array}{l}\text { Neuroactive ligand- } \\
\text { receptor interaction }\end{array}$ & 2 & $\begin{array}{l}\text { Drd2 (dopamine receptor D2) } \downarrow \text {, Drd4 (dopamine } \\
\text { receptor } D 4) \downarrow\end{array}$ \\
\hline mmu04151 & $\begin{array}{l}\text { PI3K-Akt signaling } \\
\text { pathway }\end{array}$ & 2 & $\begin{array}{l}\text { Col6a3 (collagen, type VI, alpha 3) } \downarrow \text {, Thbs } 1 \\
\text { (thrombospondin 1) } \uparrow\end{array}$ \\
\hline mmu05200 & Pathways in cancer & 2 & $\begin{array}{l}\text { Rac3 (RAS-related C3 botulinum substrate 3) } \downarrow, M m p 9 \\
\text { (matrix metallopeptidase } 9) \downarrow\end{array}$ \\
\hline
\end{tabular}

Note: $\uparrow$ indicates up-regulation in the tissue of MPFC from Reward-resilience mice compared to CUMS-resilience mice, whereas $\downarrow$ represents down-regulation. 


\begin{tabular}{|c|c|c|c|}
\hline KEGG Entry & Term & Count & Genes \\
\hline mmu01100 & $\begin{array}{l}\text { Metabolic } \\
\text { pathways }\end{array}$ & 3 & $\begin{array}{l}\text { Aldh1a2 (aldehyde dehydrogenase family } 1 \text {, } \\
\text { subfamily A2) } \uparrow \text {, Alas2 (aminolevulinic acid synthase } \\
\text { 2, erythroid) } \downarrow \text {, Gpt (glutamic pyruvic transaminase, } \\
\text { soluble) } \downarrow\end{array}$ \\
\hline mmu04919 & $\begin{array}{l}\text { Thyroid hormone } \\
\text { signaling pathway }\end{array}$ & 1 & Dio3 (deiodinase, iodothyronine type III) $\uparrow$ \\
\hline mmu04931 & Insulin resistance & 1 & $\begin{array}{l}\text { Nr1h3 (nuclear receptor subfamily } 1 \text {, group } H, \\
\text { member 3) } \uparrow\end{array}$ \\
\hline mmu00220 & $\begin{array}{l}\text { Arginine } \\
\text { biosynthesis }\end{array}$ & 1 & Gpt (glutamic pyruvic transaminase, soluble) $\downarrow$ \\
\hline mmu01200 & Carbon metabolism & 1 & Gpt (glutamic pyruvic transaminase, soluble) $\downarrow$ \\
\hline mmu04662 & $\begin{array}{l}\text { B cell receptor } \\
\text { signaling pathway }\end{array}$ & 1 & Rac3 (RAS-related C3 botulinum substrate 3) $\downarrow$ \\
\hline mmu00250 & $\begin{array}{l}\text { Alanine, aspartate } \\
\text { and glutamate } \\
\text { metabolism }\end{array}$ & 1 & Gpt (glutamic pyruvic transaminase, soluble) $\downarrow$ \\
\hline mmu05010 & Alzheimer's disease & 1 & $\begin{array}{l}\text { Atp2a } 1 \text { (ATPase, Ca + + transporting, cardiac muscle, } \\
\text { fast twitch 1) } \downarrow\end{array}$ \\
\hline mmu04360 & Axon guidance & 1 & Rac3 (RAS-related C3 botulinum substrate 3) $\downarrow$ \\
\hline mmu04810 & $\begin{array}{l}\text { Regulation of actin } \\
\text { cytoskeleton }\end{array}$ & 1 & Rac3 (RAS-related C3 botulinum substrate 3) $\downarrow$ \\
\hline mmu05212 & Pancreatic cancer & 1 & Rac3 (RAS-related C3 botulinum substrate 3) $\downarrow$ \\
\hline mmu00260 & $\begin{array}{l}\text { Glycine, serine and } \\
\text { threonine } \\
\text { metabolism }\end{array}$ & 1 & Alas2 (aminolevulinic acid synthase 2, erythroid) $\downarrow$ \\
\hline mmu04932 & $\begin{array}{l}\text { Non-alcoholic fatty } \\
\text { liver disease } \\
\text { (NAFLD) }\end{array}$ & 1 & $\begin{array}{l}\text { Nr1h3 (nuclear receptor subfamily } 1 \text {, group } H \text {, } \\
\text { member 3) } \uparrow\end{array}$ \\
\hline mmu04310 & $\begin{array}{l}\text { Wnt signaling } \\
\text { pathway }\end{array}$ & 1 & Rac3 (RAS-related C3 botulinum substrate 3) $\downarrow$ \\
\hline mmu05160 & Hepatitis C & 1 & $\begin{array}{l}\text { Nr1h3 (nuclear receptor subfamily } 1 \text {, group } H \text {, } \\
\text { member 3) } \uparrow\end{array}$ \\
\hline mmu03010 & Ribosome & 1 & Rp/37rt (ribosomal protein $L 37$, retrotransposed) $\downarrow$ \\
\hline mmu00830 & Retinol metabolism & 1 & $\begin{array}{l}\text { Aldh1a2 (aldehyde dehydrogenase family } 1, \\
\text { subfamily A2) } \uparrow\end{array}$ \\
\hline
\end{tabular}

Note: $\uparrow$ indicates up-regulation in the tissue of mPFC from Reward-resilience mice compared to CUMS-resilience mice, whereas $\downarrow$ represents down-regulation. 


\begin{tabular}{|c|c|c|c|}
\hline KEGG Entry & Term & Count & Genes \\
\hline mmu05412 & $\begin{array}{l}\text { Arrhythmogenic } \\
\text { right ventricular } \\
\text { cardiomyopathy } \\
\text { (ARVC) }\end{array}$ & 1 & Pkp2 (plakophilin 2) $\downarrow$ \\
\hline mmu04022 & $\begin{array}{l}\text { cGMP-PKG } \\
\text { signaling pathway }\end{array}$ & 1 & $\begin{array}{l}\text { Atp2a1 (ATPase, Ca + + transporting, cardiac muscle, } \\
\text { fast twitch 1) } \downarrow\end{array}$ \\
\hline mmu04664 & $\begin{array}{l}\text { Fc epsilon RI } \\
\text { signaling pathway }\end{array}$ & 1 & Rac3 (RAS-related C3 botulinum substrate 3) $\downarrow$ \\
\hline mmu05202 & $\begin{array}{l}\text { Transcriptional } \\
\text { misregulation in } \\
\text { cancer }\end{array}$ & 1 & Mmp9 (matrix metallopeptidase 9) $\downarrow$ \\
\hline mmu01230 & $\begin{array}{l}\text { Biosynthesis of } \\
\text { amino acids }\end{array}$ & 1 & Gpt (glutamic pyruvic transaminase, soluble) $\downarrow$ \\
\hline mmu04370 & $\begin{array}{l}\text { VEGF signaling } \\
\text { pathway }\end{array}$ & 1 & Rac3 (RAS-related C3 botulinum substrate 3) $\downarrow$ \\
\hline mmu05161 & Hepatitis B & 1 & Mmp9 (matrix metallopeptidase 9) $\downarrow$ \\
\hline mmu05144 & Malaria & 1 & Thbs1 (thrombospondin 1) $\uparrow$ \\
\hline mmu04350 & $\begin{array}{l}\text { TGF-beta signaling } \\
\text { pathway }\end{array}$ & 1 & Thbs1 (thrombospondin 1) 个 \\
\hline mmu05231 & $\begin{array}{l}\text { Choline } \\
\text { metabolism in } \\
\text { cancer }\end{array}$ & 1 & Rac3 (RAS-related C3 botulinum substrate 3) $\downarrow$ \\
\hline mmu04621 & $\begin{array}{l}\text { NOD-like receptor } \\
\text { signaling pathway }\end{array}$ & 1 & $\begin{array}{l}\text { Pstpip } 1 \text { (proline-serine-threonine phosphatase- } \\
\text { interacting protein 1) } \downarrow\end{array}$ \\
\hline mmu04960 & $\begin{array}{l}\text { Aldosterone- } \\
\text { regulated sodium } \\
\text { reabsorption }\end{array}$ & 1 & Sfn (stratifin) $\downarrow$ \\
\hline mmu05416 & Viral myocarditis & 1 & Rac3 (RAS-related C3 botulinum substrate 3) $\downarrow$ \\
\hline mmu00860 & $\begin{array}{l}\text { Porphyrin and } \\
\text { chlorophyll } \\
\text { metabolism }\end{array}$ & 1 & Alas2 (aminolevulinic acid synthase 2, erythroid) $\downarrow$ \\
\hline mmu04540 & Gap junction & 1 & Drd2 (dopamine receptor D2) $\downarrow$ \\
\hline mmu04145 & Phagosome & 1 & Thbs1 (thrombospondin 1) $\uparrow$ \\
\hline mmu04020 & $\begin{array}{l}\text { Calcium signaling } \\
\text { pathway }\end{array}$ & 1 & $\begin{array}{l}\text { Atp2a } 1 \text { (ATPase, Ca + + transporting, cardiac muscle, } \\
\text { fast twitch 1) } \downarrow\end{array}$ \\
\hline mmu04520 & Adherens junction & 1 & Rac3 (RAS-related C3 botulinum substrate 3) $\downarrow$ \\
\hline
\end{tabular}




\begin{tabular}{|c|c|c|c|}
\hline KEGG Entry & Term & Count & Genes \\
\hline mmu05012 & Parkinson's disease & 1 & Drd2 (dopamine receptor D2) $\downarrow$ \\
\hline mmu04650 & $\begin{array}{l}\text { Natural killer cell } \\
\text { mediated } \\
\text { cytotoxicity }\end{array}$ & 1 & Rac3 (RAS-related C3 botulinum substrate 3) $\downarrow$ \\
\hline mmu04668 & $\begin{array}{l}\text { TNF signaling } \\
\text { pathway }\end{array}$ & 1 & Mmp9 (matrix metallopeptidase 9) $\downarrow$ \\
\hline mmu04670 & $\begin{array}{l}\text { Leukocyte } \\
\text { transendothelial } \\
\text { migration }\end{array}$ & 1 & Mmp9 (matrix metallopeptidase 9) $\downarrow$ \\
\hline mmu04110 & Cell cycle & 1 & Sfn (stratifin) $\downarrow$ \\
\hline mmu04514 & $\begin{array}{l}\text { Cell adhesion } \\
\text { molecules (CAMs) }\end{array}$ & 1 & Mpz (myelin protein zero) $\uparrow$ \\
\hline mmu04972 & $\begin{array}{l}\text { Pancreatic } \\
\text { secretion }\end{array}$ & 1 & $\begin{array}{l}\text { Atp2a } 1 \text { (ATPase, Ca ++ transporting, cardiac muscle, } \\
\text { fast twitch 1) } \downarrow\end{array}$ \\
\hline mmu01210 & $\begin{array}{l}\text { 2-Oxocarboxylic } \\
\text { acid metabolism }\end{array}$ & 1 & Gpt (glutamic pyruvic transaminase, soluble) $\downarrow$ \\
\hline mmu04071 & $\begin{array}{l}\text { Sphingolipid } \\
\text { signaling pathway }\end{array}$ & 1 & Rac3 (RAS-related C3 botulinum substrate 3) $\downarrow$ \\
\hline mmu05030 & Cocaine addiction & 1 & Drd2 (dopamine receptor D2) $\downarrow$ \\
\hline mmu03320 & $\begin{array}{l}\text { PPAR signaling } \\
\text { pathway }\end{array}$ & 1 & $\begin{array}{l}\text { Nr1h3 (nuclear receptor subfamily } 1 \text {, group } H, \\
\text { member 3) } \uparrow\end{array}$ \\
\hline mmu04010 & $\begin{array}{l}\text { MAPK signaling } \\
\text { pathway }\end{array}$ & 1 & Rac3 (RAS-related C3 botulinum substrate 3) $\downarrow$ \\
\hline mmu04014 & $\begin{array}{l}\text { Ras signaling } \\
\text { pathway }\end{array}$ & 1 & Rac3 (RAS-related C3 botulinum substrate 3) $\downarrow$ \\
\hline mmu04915 & $\begin{array}{l}\text { Estrogen signaling } \\
\text { pathway }\end{array}$ & 1 & Mmp9 (matrix metallopeptidase 9) $\downarrow$ \\
\hline mmu05034 & Alcoholism & & Drd2 (dopamine receptor D2) $\downarrow$ \\
\hline
\end{tabular}

Furthermore, to validate the sequencing data above, we ran quantitative RT-PCR (qRT-PCR) from tissues that were used for mRNA sequencing. 10 mRNAs were selected to conduct qRT-PCR, the expressions of Artn, Dio3, Klf4, Pcdha1, Pcdhgb4, and Slc6a13 are increased, as well as the expressions of Col6a3, Drd2, Mmp9, and Rac3 are decreased (Figure S3). Consistent results achieved by mRNA sequencing and qRTPCR confirm the validation of our study.

Accompanying with companion altered miRNA expression in resilience mice. 
Studies have revealed that patients with psychiatric disorders have altered miRNA expression profiles in the brain and circulation, and multiple roles of miRNAs in psychiatric disorders have summarized [51]. To investigate the impact of accompanying with companion on miRNA, we performed differential expression analysis of miRNA by high-throughput sequencing. As shown in Table S8, compared with the CUMSresilience mice, a total of 61 differentially expressed miRNAs were up-regulated and 135 differentially expressed miRNAs were down-regulated in the Reward-resilience mice. In order to validate the finding by sequencing miRNA analysis, quantitative RT-PCR were performed for certain miRNAs. As shown in Figure S4, qRT-PCR analysis results for miR-149-5p, miR-183-5p, miR-199b-3p, miR-323-5p, miR-337-3p, miR-384$5 p$, miR-451a, miR-484, miR-671-5p, and miR-702-3p were consistent with miRNA sequencing results in Reward-resilience versus CUMS-resilience mice.

miRNAs can act as an 'expression switch' and block the expression of their target genes [52]. In this study, if the mRNAs downregulated were caused by miRNAs, their correspondent miRNAs should be upregulated, or vice versa. Based on three databases (RNAhybrid, Targetscan and miRanda), the target genes of miRNAs were predicted and matched with the mRNA measured by mRNA sequencing to form the complex interactions between miRNAs and mRNAs (Tables 3 and 4). As shown in Fig. 4a and 4b, 63 miRNAs established regulatory relationships with 34 mRNAs in CUMS-resilience versus Reward-resilience mice. Furthermore, the miRNAs targeted mRNAs were validated by qRT-PCR and dual luciferase reporter assay. As shown in Fig. 4c and 4d, there were inverse correlations between Pcdha 1 and miRNA-337-3p, as well as between Rac3 and miRNA-671-5p from qRT-PCR analysis. In dual luciferase report assay, the relative activities of luciferase reporter for $P c d h a 1$ and $R a c 3$ are significantly decreased by the mimics of miRNA-337-3p and miRNA-671-5p respectively, but not negative control (Fig. 4e and 4f). These reductions can be reversed by changing the binding sites of miRNA-337-3p and miRNA-671-5p in Pcdha1 and Rac3. These results support that mRNA Pcdha 1 is the direct target of miRNA-337-3p, and mRNA Rac3 is the direct target of miRNA-671-5p, which is consistent with our bioinformatics analyses in the prediction of miRNA target genes. 
Table 3

Differently expression miRNAs predict target mRNAs in CUMS-resilience versus Reward-resilience.

\section{miRNAs The predicted target mRNAs that match DEGs in transcriptome *}

miR- Aldh1a2 $\uparrow$, Klf4 $\uparrow$

1231-

$3 \mathrm{p} \downarrow$

miR- $\quad A l x 3 \uparrow, M p z \uparrow$

$1249-$

$5 \mathrm{p} \downarrow$

miR- $\quad$ Adam331

$1291 \downarrow$

miR- Obscn $\downarrow$

$141-$

$5 \mathrm{p} \uparrow$

miR- $\quad$ Adam33个

$146 b-$

$3 \mathrm{p} \downarrow$

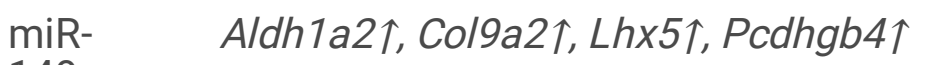

$149-$

$5 \mathrm{p} \downarrow$

miR- $\quad P l k 5 \uparrow$

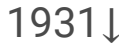

miR- Adamts1个

1934-

$5 \mathrm{p} \downarrow$

miR- Crispld2 $\uparrow$

196a-

$5 \mathrm{p} \downarrow$

miR- Crispld $2 \uparrow$

196b-

$5 \mathrm{p} \downarrow$

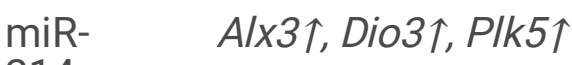

214-

$3 p \downarrow$

miR-24- $\quad L h \times 5 \uparrow$

$3 p \downarrow$

miR-

$26 b-$

$3 p \downarrow$
miRNAs The predicted target mRNAs that match DEGs in transcriptome *

miR- $\quad$ Cyr61个

5617-

$3 p \downarrow$

miR-

652-

$5 \mathrm{p} \downarrow$

miR-

$6538 \downarrow$

miR-

Obscn\

$669 \mathrm{~m}-$

$5 \mathrm{p} \uparrow$

miR-

$670-$

$5 \mathrm{p} \uparrow$

miR-

671-

$5 \mathrm{p} \uparrow$

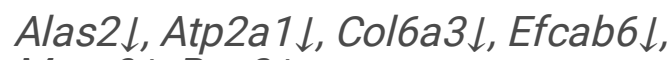

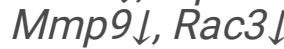

miR-

6919-

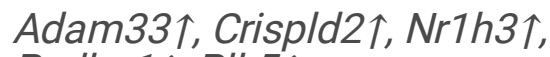

$3 p \downarrow$

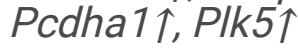

miR-

6919-

Col6a3 $\downarrow$, Obscn $\downarrow, P k p 2 \downarrow$

$5 \mathrm{p} \uparrow$

miR-

6954-

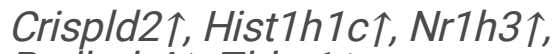

$5 \mathrm{p} \downarrow$

miR-

6982-

$5 \mathrm{p} \downarrow$

miR-

698-

$5 \mathrm{p} \downarrow$

miR- Pcdha1个

6997-

$5 \mathrm{p} \downarrow$

miR-

6999-

$5 \mathrm{p} \uparrow$

Note: $\uparrow$ indicates up-regulation in the tissue of mPFC from Reward-resilience mice compared to CUMS-resilience mice, whereas $\downarrow$ represents down-regulation. 


\begin{tabular}{|c|c|c|c|}
\hline miRNAs & $\begin{array}{l}\text { The predicted target mRNAs that } \\
\text { match DEGs in transcriptome * }\end{array}$ & miRNAs & $\begin{array}{l}\text { The predicted target mRNAs that } \\
\text { match DEGs in transcriptome * }\end{array}$ \\
\hline $\begin{array}{l}\text { miR- } \\
299 a- \\
3 p \uparrow\end{array}$ & Col6a3\ & $\begin{array}{l}\text { miR- } \\
7004- \\
5 p \downarrow\end{array}$ & 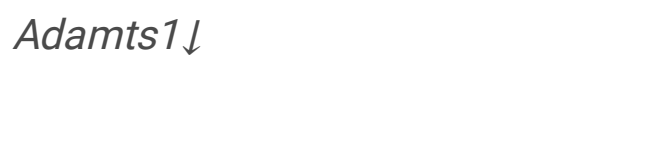 \\
\hline $\begin{array}{l}\text { miR- } \\
3078- \\
5 p \downarrow\end{array}$ & $M p z \uparrow$ & $\begin{array}{l}\text { miR- } \\
7019- \\
3 p \downarrow\end{array}$ & 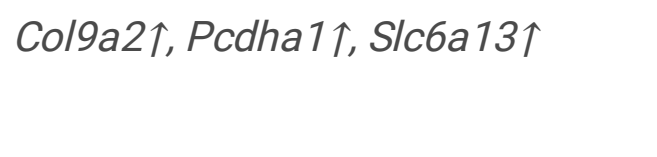 \\
\hline $\begin{array}{l}\text { miR- } \\
3087- \\
5 p \downarrow\end{array}$ & 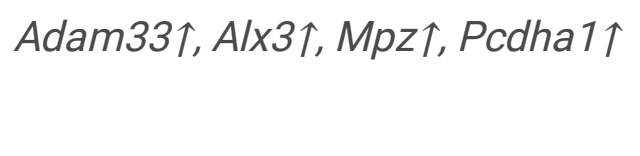 & $\begin{array}{l}\text { miR- } \\
702- \\
3 p \downarrow\end{array}$ & 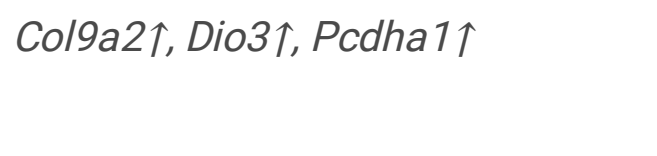 \\
\hline $\begin{array}{l}\text { miR- } \\
3093- \\
5 p \downarrow\end{array}$ & 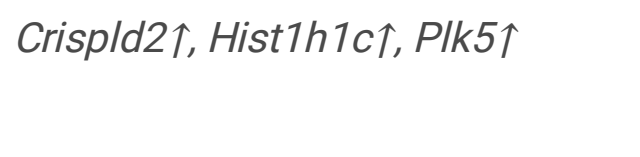 & $\begin{array}{l}\text { miR- } \\
7032- \\
5 p \downarrow\end{array}$ & 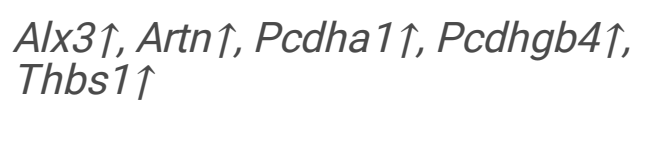 \\
\hline $\begin{array}{l}\text { miR- } \\
323- \\
5 p \downarrow\end{array}$ & Artn $\uparrow, K I f 4 \uparrow$ & $\begin{array}{l}\text { miR- } \\
7043- \\
3 p \uparrow\end{array}$ & Obscn $\downarrow$ \\
\hline $\begin{array}{l}\text { miR- } \\
328- \\
5 p \downarrow\end{array}$ & 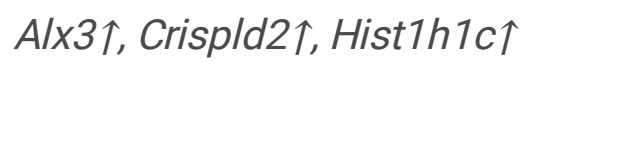 & $\begin{array}{l}\text { miR- } \\
7052- \\
3 p \downarrow\end{array}$ & 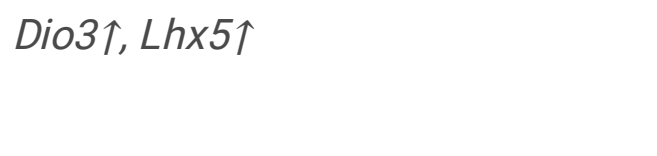 \\
\hline $\begin{array}{l}\text { miR- } \\
337- \\
3 p \downarrow\end{array}$ & 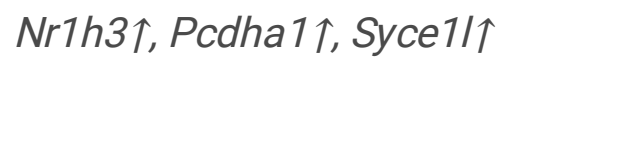 & $\begin{array}{l}\text { miR- } \\
7053- \\
3 p \downarrow\end{array}$ & 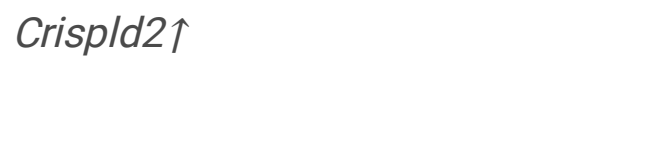 \\
\hline $\begin{array}{l}\text { miR- } \\
339- \\
5 p \downarrow\end{array}$ & 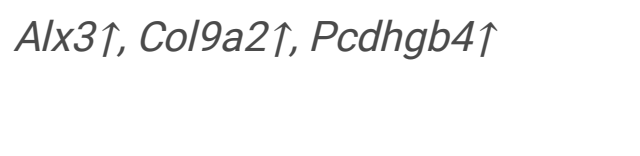 & $\begin{array}{l}\text { miR- } \\
7054- \\
3 p \downarrow\end{array}$ & $L h \times 5 \uparrow$ \\
\hline $\begin{array}{l}\text { miR- } \\
341- \\
5 p \downarrow\end{array}$ & 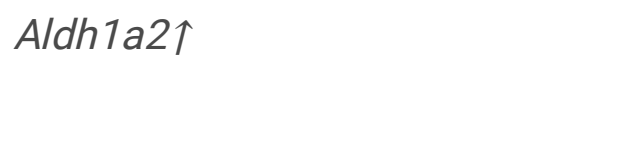 & $\begin{array}{l}\text { miR- } \\
7056- \\
3 p \downarrow\end{array}$ & Col9a2 $\uparrow$ \\
\hline $\begin{array}{l}\text { miR- } \\
3544- \\
3 p \downarrow\end{array}$ & 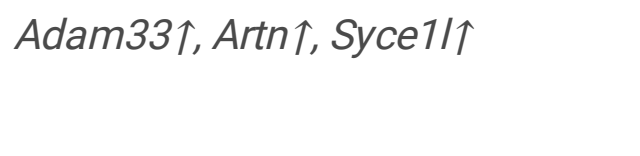 & $\begin{array}{l}\text { miR- } \\
7070- \\
3 p \downarrow\end{array}$ & 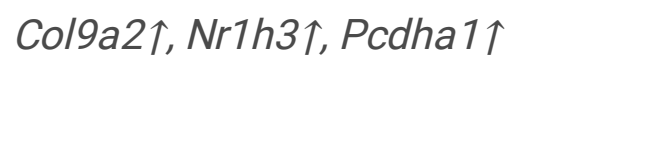 \\
\hline $\begin{array}{l}\text { miR- } \\
378 d \uparrow\end{array}$ & Col6a3 $\downarrow$, Obscn $\downarrow, P k p 2 \downarrow$ & $\begin{array}{l}\text { miR- } \\
7071- \\
5 p \downarrow\end{array}$ & 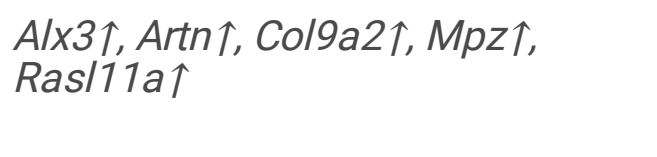 \\
\hline $\begin{array}{l}\text { miR- } \\
449 \mathrm{c}- \\
5 \mathrm{p} \uparrow\end{array}$ & Obscn $\downarrow$ & $\begin{array}{l}\text { miR- } \\
7115- \\
5 p \downarrow\end{array}$ & 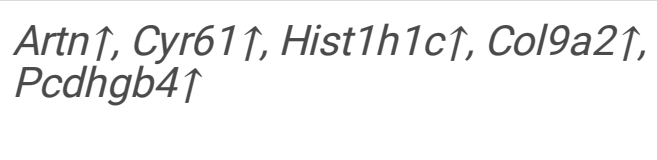 \\
\hline $\begin{array}{l}\text { miR- } \\
466 \mathrm{~h}- \\
5 \mathrm{p} \uparrow\end{array}$ & 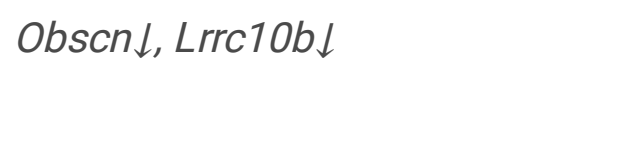 & $\begin{array}{l}\text { miR- } \\
7235- \\
3 p \uparrow\end{array}$ & Efcab3个 \\
\hline
\end{tabular}

Note: $\uparrow$ indicates up-regulation in the tissue of mPFC from Reward-resilience mice compared to CUMS-resilience mice, whereas $\downarrow$ represents down-regulation. 


\begin{tabular}{|c|c|c|c|}
\hline miRNAs & $\begin{array}{l}\text { The predicted target mRNAs that } \\
\text { match DEGs in transcriptome * }\end{array}$ & miRNAs & $\begin{array}{l}\text { The predicted target mRNAs that } \\
\text { match DEGs in transcriptome * }\end{array}$ \\
\hline $\begin{array}{l}\operatorname{miR}- \\
484 \downarrow\end{array}$ & 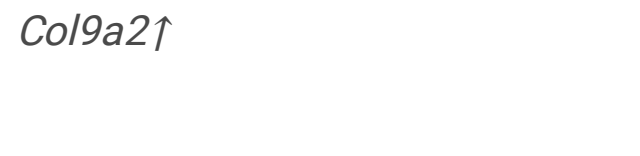 & $\begin{array}{l}\text { miR- } \\
758- \\
5 p \downarrow\end{array}$ & Col9a2؟ \\
\hline $\begin{array}{l}\text { miR- } \\
486 b- \\
5 p \uparrow\end{array}$ & 4933409K07Rik $\downarrow$ & $\begin{array}{l}\text { miR- } \\
760- \\
3 p \downarrow\end{array}$ & 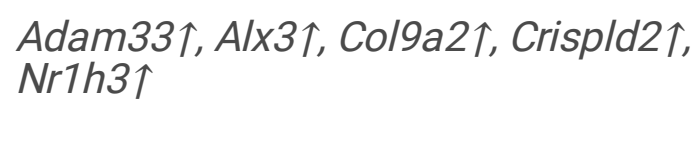 \\
\hline $\begin{array}{l}\text { miR- } \\
503- \\
5 p \uparrow\end{array}$ & Efcab6 $\downarrow$, Obscn $\downarrow$ & $\begin{array}{l}\text { miR- } \\
770- \\
3 p \downarrow\end{array}$ & Adam33个 \\
\hline $\begin{array}{l}\text { miR- } \\
5126 \downarrow\end{array}$ & 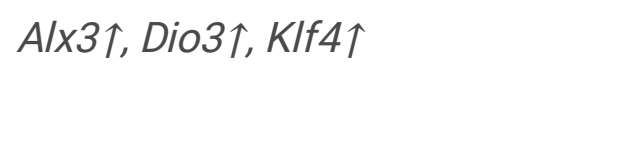 & $\begin{array}{l}\text { miR- } \\
873 a- \\
5 p \uparrow\end{array}$ & Obscn $\downarrow$ \\
\hline $\begin{array}{l}\text { miR- } \\
5129- \\
5 p \uparrow\end{array}$ & Obscn $\downarrow$ & $\begin{array}{l}\text { miR- } \\
873 a- \\
5 p \uparrow\end{array}$ & Tmem40\} $\\
$\hline
\end{tabular}


Table 4

Differently expression mRNAs predict target miRNAs in CUMS-resilience versus Reward-resilience.

$\begin{array}{llll}\begin{array}{l}\text { Gene } \\ \text { Symbol }\end{array} & \begin{array}{l}\text { The predicted target miRNAs that match } \\ \text { DEGs in transcriptome* }\end{array} & \begin{array}{l}\text { Gene } \\ \text { Symbol }\end{array} & \begin{array}{l}\text { The predicted target } \\ \text { miRNAs that match DEGs } \\ \text { in transcriptome* }\end{array}\end{array}$

Adam33 $\uparrow \quad$ miR-1291 $\downarrow$, miR-146b-3p $\downarrow$, miR-3087-5p $\downarrow, \quad L$ Lrc10b $\downarrow \quad$ miR-466p-3p $\uparrow$ miR-3544-3p $\downarrow$, miR-6919-3p $\downarrow$, miR-760-

$3 p \downarrow$, miR-770-3p $\downarrow$

Adamts1个 miR-1934-5p $\downarrow$, miR-7004-5p $\downarrow \quad M m p 9 \downarrow \quad m i R-671-5 p \uparrow$

Alas2 $\downarrow \quad m i R-671-5 p \uparrow \quad M p z \uparrow \quad m i R-1249-5 p \downarrow$, mmiR-3078-

$5 \mathrm{p} \downarrow, \mathrm{miR}-3087-5 \mathrm{p} \downarrow, \mathrm{miR}-$

$6982-5 p \downarrow$, miR-7071-5p $\downarrow$

Aldh1a2 $\uparrow \quad \operatorname{miR}-1231-3 p \downarrow$, miR-149-5p $\downarrow$, miR-341-5p $\downarrow \quad N r 1 h 3 \uparrow$

miR-337-3p $\downarrow$, miR-652-5p $\downarrow$, miR-6919-3p $\downarrow$, miR-6954-

$5 \mathrm{p} \downarrow$, miR-7070-3p $\downarrow$, miR-

$760-3 p \downarrow$

$A / x 3 \uparrow$

miR-1249-5p $\downarrow$, miR-214-3p $\downarrow$, miR-3087-

$5 \mathrm{p} \downarrow$, miR-328-5p $\downarrow$, miR-339-5p $\downarrow$, miR-

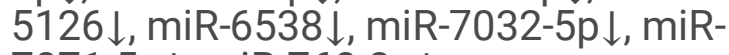

7071-5p $\downarrow$, miR-760-3p $\downarrow$

Obscn $\downarrow$

miR-141-5p $\uparrow, m i R-378 d \uparrow$, miR-449c-5p $\uparrow$, miR-466 h$5 \mathrm{p} \uparrow, \mathrm{miR}-503-5 \mathrm{p} \uparrow, \mathrm{miR}-$ 5129-5p $\uparrow, m i R-669 m-5 p \uparrow$, miR-670-5p $\uparrow$, miR-6919$5 \mathrm{p} \uparrow, \mathrm{miR}-7043-3 \mathrm{p} \uparrow, \mathrm{miR}-$ $873 a-5 p \uparrow$

Artn $\uparrow \quad m i R-323-5 p \downarrow$, miR-3544-3p $\downarrow$, miR-6538 $\downarrow$, miR-7032-5p $\downarrow$, mmu-miR-7071-5p $\downarrow$, miR-

Pcdha1个

miR-3087-5p $\downarrow$, miR-337-

7115-5p $\downarrow$ $3 p \downarrow$, miR-6919-3p $\downarrow$, miR6997-5p $\downarrow$, miR-7019-3p $\downarrow$, miR-702-3p $\downarrow$, miR-7032$5 p \downarrow, \operatorname{miR}-7070-3 p \downarrow$

Atp2a1 $\downarrow$ miR-671-5p $\uparrow$

Pcdhgb4 $\uparrow$

miR-149-5p $\downarrow$, miR-339-5p $\downarrow$, miR-6954-5p $\downarrow$, miR-6982$5 \mathrm{p} \downarrow$, miR-7032-5p $\downarrow$, miR7117-5p $\downarrow$

Col6a3 $\downarrow$

$m i R-299 a-3 p \uparrow, m i R-378 d \uparrow, m i R-671-5 p \uparrow$, miR-6919-5p $\uparrow$

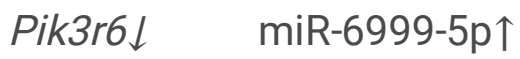

Col9a2

miR-149-5p $\downarrow$, miR-339-5p $\downarrow$, miR-484 $\downarrow$, miR6982-5p $\downarrow$, miR-7019-3p $\downarrow$, -miR-702-3p $\downarrow$,

$P k p 2 \downarrow$ miR-378d $\uparrow$, miR-6919-5p $\uparrow$ miR-702-3p $\downarrow$, miR-7056-3p $\downarrow$, miR-7070$3 p \downarrow$, miR-7071-5p $\downarrow$, miR-7117-5p $\downarrow$, miR-

758-5p $\downarrow$, miR-760-3p $\downarrow$

Crispld $2 \uparrow$

miR-196a-5p $\downarrow$, miR-196b-5p $\downarrow$, miR-3093-

5p $\downarrow$, miR-328-5p $\downarrow$, miR-652-5p $\downarrow$, miR-6919-

Plk5

$3 p \downarrow$, miR-6954-5p $\downarrow$, miR-698-5p $\downarrow$, miR-

7053-3p $\downarrow$, miR-760-3p $\downarrow$

Cyr61个

miR-5617-3p $\downarrow$, miR-7115-5p $\downarrow$

Rac3\

miR-671-5p $\uparrow$

Note: $\uparrow$ indicates up-regulation in the tissue of mPFC from Reward-resilience mice compared to CUMS-resilience mice, whereas $\downarrow$ represents down-regulation. 


\begin{tabular}{|c|c|c|c|}
\hline $\begin{array}{l}\text { Gene } \\
\text { Symbol }\end{array}$ & $\begin{array}{l}\text { The predicted target miRNAs that match } \\
\text { DEGs in transcriptome * }\end{array}$ & $\begin{array}{l}\text { Gene } \\
\text { Symbol }\end{array}$ & $\begin{array}{l}\text { The predicted target } \\
\text { miRNAs that match DEGs } \\
\text { in transcriptome* }\end{array}$ \\
\hline Dio3个 & $\begin{array}{l}\text { miR-214-3p } \downarrow \text {, miR-5126 } \downarrow \text {, miR-6538 } \downarrow \text {, miR- } \\
\text { 702-3p } \downarrow \text {, miR-7052-3p } \downarrow\end{array}$ & Rasl11a个 & $\begin{array}{l}\text { miR-6982-5p } \downarrow \text {, miR-7071- } \\
5 p \downarrow\end{array}$ \\
\hline Efcab3\} $&{\operatorname{miR}-7235-3 p \uparrow} &{\text { Slc6а13个 }} &{\begin{array}{l}\operatorname{miR}-26 b-3 p \downarrow, \text { miR-7019- } \\
3 p \downarrow\end{array}} \\
{\hline \text { Efcab6】 }} &{\text { miR-503-5p } \uparrow, \text { miR-671-5p } \uparrow} &{\text { Syce1/个 }} &{\begin{array}{l}\operatorname{miR}-337-3 p \downarrow, \text { miR-3544- } \\
3 p \downarrow\end{array}} \\
{\hline \text { Hist1h1c }} &{\begin{array}{l}\text { miR-3093-5p } \downarrow \text {, miR-328-5p } \downarrow \text {, miR-6538 } \downarrow \text {, } \\
\text { miR-6954-5p } \downarrow \text {, miR-7115-5p } \downarrow\end{array}} &{\text { Thbs1个 }} &{\begin{array}{l}\operatorname{miR}-6954-5 p \downarrow \text {, miR-7032- } \\
5 p \downarrow\end{array}} \\
{\hline \text { KIf4个 }} &{\text { miR-1231-3p } \downarrow \text {, miR-323-5p } \downarrow \text {, miR-5126 } \downarrow} &{\text { Tmem40\} } &{\operatorname{miR}-873 a-5 p \uparrow} \\
{\hline L h \times 5 \uparrow} &{\begin{array}{l}\text { miR-149-5p } \downarrow \text {, miR-24-3p } \downarrow \text {, miR-7052-3p } \downarrow \text {, } \\
\text { miR-7054-3p } \downarrow\end{array}} &{ } &{ } \\
\hline\end{array}$
\end{tabular}

\section{Discussion}

Research suggests that major depressive disorder emerges out of a three-pronged series of interacting impairments in the domains of stress regulation, reward and mentalizing [53]. The motivation induced by reward can effectively promote individual's emotional regulation ability [54], and lack of rewards in life can cause the brain's reward circuits to be not sufficiently activated or actively used, which may reduce the function of the brain's reward circuits $[7,8]$. Studies have shown that encouraging patients to participate in reward activities during treatment has been found to be effective in alleviating MDD [27]. In this study, we add a natural reward (companion) on the basic of CUMS model to explore the effect of reward treatment on CUMS-induced depression-like behaviors. Behavioral results showed that accompanying with companion ameliorated depression-like behaviors induced by CUMS treatments. Subsequently, the high-throughput sequencing for both mRNAs and miRNAs were performed to gain indepth insights into the molecular function. Excitingly, we screened a large number of genes associated with depression-like behaviors, resilience and accompanying with companion.

Depression is a heterogeneous, highly prevalent and moderately inherited disease. In addition, environmental factors, such as sexual, physical or emotional abuse during childhood, are strongly associated with the risk of developing MDD [55]. Therefore, the etiology of MDD is multifactorial, and no established mechanism can explain all aspects of this disease [55]. In this study, two depression-like behavior mice derived from CUMS and reward treatments, although they are phenotypically consistent, there are still large differences in gene expression in the mPFC, which provides an evidence for the different mechanisms of depression in different environments. Nevertheless, we screened 45 coexpressed genes from the mPFC of two depression-like behavior mice, which were defined as DEGs 
associated with depression-like behaviors. Subsequent KEGG pathway analysis found that these genes were mainly enriched to Metabolic pathways, cAMP signaling pathway, ECM-receptor interaction, PI3KAkt signaling pathway, Serotonergic synapse and Neuroactive ligand-receptor interaction. How important these genes are to preventing and treating depression, more research is needed in the future. Generally, most individuals may not suffer from major depression by chronic stress, i.e., resilience [50], implying the presence of endogenous anti-depression in the brain. In this study, we also found resilience mice under the CUMS and CUMS with accompanying with companion, respectively. Compared to 45 DEGs associated with depression-like behaviors, only 8 common DEGs were obtained between control versus CUMS-resilience and control versus Reward-resilience comparisons, which indicated that although resilience derived from CUMS and reward treatments are consistent in behaviors, there are still significant differences in molecular changes in the prefrontal brain. Therefore, different antidepressant measures and inherent genetic factors, different antidepressant molecular mechanisms may appear.

Recent studies have shown that low perceived social support and loneliness were related to depression course and recovery $[56,57]$, and only the social support characteristic of few negative experiences with the support from a partner or close friend, and limited feelings of loneliness proved to have unique predictive value for a favorable course of depression [56]. Therefore, it's a great interest to test whether a partner or close friend can contribute to better recovery and prevention of MDD. In our study, mice treated by CUMS and the companion used for accompanying are from the same litter, which is a good way to simulate a partner or close friend. What is more, accompanying with companion achieved a certain effect in ameliorating CUMS-induced depression-like behaviors, and the molecular mechanism were in-depth investigated. Associated with accompanying with companion, 59 mRNAs and 196 miRNAs were identified and used to establish the miRNA/mRNA network. Noteworthy, in CUMS-resilience versus Reward-resilience, accompanying with companion disrupted the expression of dopamine receptors (Drd2 and Drd4), which are enrich in dopaminergic synapse and neuroactive ligand-receptor interaction pathways.

\section{Conclusions}

Taken together, our present study by analyzing miRNA and mRNA profiles provides numerous potential drug targets for depression and comprehensive view about molecular mechanisms underlying reward treatment ameliorating depressive-like behaviors, which giving better theoretical support for the application of reward intervention in the treatment of depression.

\section{Abbreviations}

MDD: Major depressive disorder; mRNA: Messenger RNA; miRNA: MicroRNA SPT: Sucrose preference test; YMT: Y-maze Test; FST: Forced swimming test; BGI: Beijing Genomics Institute; RPKM: Reads per kilobase per million; DEGs: Differentially expressed genes; qRT-PCR: Quantitative real-time RT-PCR; CUMS: Chronic unpredictable mild stress; KEGG: Kyoto Encyclopedia of Genes and Genomes 


\section{Declarations}

\section{Ethics approval and consent to participate:}

Experiments were performed in accordance with the guideline and regulation by Administration Office of Laboratory Animals at Beijing China, in which all Protocols were approved by Institutional Animal Care and Use Committee in this office (B10831).

\section{Consent for publication:}

Not applicable.

\section{Availability of data and materials:}

The datasets supporting the conclusions of this article are included within the article and its additional files.

\section{Competing interests:}

All authors declare no competing interest.

\section{Funding:}

This study is supported by the National Natural Science Foundation of China (31801953) to Zhenhua Song. These funding were role in the analysis of data and in writing the manuscript. Zhenhua Song contributed to experiments and data analyses.

\section{Authors' contributions:}

T An and Z Song contributed to experiments and data analyses. J-H Wang contributed to concept, project design and paper writing. All authors have read and approved the final version of the manuscript.

\section{Acknowledgement:}

The authors wish to thanks all of the participants of the study. 


\section{Authors' Information:}

${ }^{1}$ Qingdao University, School of Pharmacy, 38 Dengzhou, Qingdao.

${ }^{2}$ University of Chinese Academy of Sciences, 15 Datun Road, Chaoyang District.

\section{References}

1. Kessler RC, Berglund P, Demler O, Jin R, Koretz D, Merikangas KR, Rush AJ, Walters EE, Wang PS, National Comorbidity Survey R. The epidemiology of major depressive disorder: results from the National Comorbidity Survey Replication (NCS-R). JAMA. 2003;289(23):3095-105.

2. Camp NJ, Cannon-Albright LA. Dissecting the genetic etiology of major depressive disorder using linkage analysis. Trends Mol Med. 2005;11(3):138-44.

3. Murrough JW, lacoviello B, Neumeister A, Charney DS, losifescu DV. Cognitive dysfunction in depression: neurocircuitry and new therapeutic strategies. Neurobiol Learn Mem. 2011;96(4):553-63.

4. Hamilton JP, Chen MC, Gotlib IH. Neural systems approaches to understanding major depressive disorder: an intrinsic functional organization perspective. Neurobiol Dis. 2013;52:4-11.

5. Battle DE. Diagnostic and Statistical Manual of Mental Disorders (DSM). Codas. 2013;25(2):191-2.

6. Lin SH, Chou ML, Chen WC, Lai YS, Lu KH, Hao CW, Sheen LY. A medicinal herb, Melissa officinalis L. ameliorates depressive-like behavior of rats in the forced swimming test via regulating the serotonergic neurotransmitter. J Ethnopharmacol. 2015;175:266-72.

7. Wang JH, Cui S. Associative memory cells: Formation, function and perspective. F1000Research. 2017;6:283.

8. Zhu Z, Wang G, Ma K, Cui S, Wang JH. GABAergic neurons in nucleus accumbens are correlated to resilience and vulnerability to chronic stress for major depression. Oncotarget. 2017;8(22):35933-45.

9. Day JJ, Carelli RM. The nucleus accumbens and Pavlovian reward learning. Neuroscientist. 2007;13(2):148-59.

10. Kim CK, Ye L, Jennings JH, Pichamoorthy N, Tang DD, Yoo AW, Ramakrishnan C, Deisseroth K. Molecular and Circuit-Dynamical Identification of Top-Down Neural Mechanisms for Restraint of Reward Seeking. Cell. 2017;170(5):1013-27 e1014.

11. Ikemoto S. Dopamine reward circuitry: two projection systems from the ventral midbrain to the nucleus accumbens-olfactory tubercle complex. Brain Res Rev. 2007;56(1):27-78.

12. Grabenhorst F, Rolls ET. Value, pleasure and choice in the ventral prefrontal cortex. Trends Cogn Sci. 2011;15(2):56-67. 
13. Gong L, Yin Y, He C, Ye Q, Bai F, Yuan Y, Zhang H, Lv L, Zhang H, Xie C, et al. Disrupted reward circuits is associated with cognitive deficits and depression severity in major depressive disorder. J Psychiatr Res. 2017;84:9-17.

14. Eshel N, Roiser JP. Reward and punishment processing in depression. Biol Psychiatry. 2010;68(2):118-24.

15. Roiser J, Farmer A, Lam D, Burke A, O'Neill N, Keating S, Smith GP, Sahakian B, McGuffin P. The effect of positive mood induction on emotional processing in euthymic individuals with bipolar disorder and controls. Psychological medicine. 2009;39(5):785-91.

16. Wise RA. Drug-activation of brain reward pathways. Drug Alcohol Depend. 1998;51(1-2):13-22.

17. Koob GF, Le Moal M. Drug addiction, dysregulation of reward, and allostasis. Neuropsychopharmacology: official publication of the American College of Neuropsychopharmacology. 2001;24(2):97-129.

18. Radwan GN, El-Setouhy M, Mohamed MK, Hamid MA, Azem SA, Kamel O, Israel E, Loffredo CA. DRD2/ANKK1 Taql polymorphism and smoking behavior of Egyptian male cigarette smokers. Nicotine tobacco research: official journal of the Society for Research on Nicotine Tobacco. 2007;9(12):1325-9.

19. da Silva Lobo DS, Vallada HP, Knight J, Martins SS, Tavares H, Gentil V, Kennedy JL. Dopamine genes and pathological gambling in discordant sib-pairs. Journal of gambling studies. 2007;23(4):421-33.

20. Russo SJ, Nestler EJ. The brain reward circuitry in mood disorders. Nature reviews Neuroscience. 2013;14(9):609-25.

21. Koelsch S. Brain correlates of music-evoked emotions. Nature reviews Neuroscience. 2014;15(3):170-80.

22. Reybrouck M, Eerola T. Music and Its Inductive Power: A Psychobiological and Evolutionary Approach to Musical Emotions. Front Psychol 2017, 8(1664 - 1078 (Print)):494.

23. Juslin PN, Vastfjall D. Emotional responses to music: the need to consider underlying mechanisms. Behav Brain Sci. 2008;31(5):559-75. discussion 575-621.

24. Braun Janzen T, Al Shirawi MI, Rotzinger S, Kennedy SH, Bartel L. A Pilot Study Investigating the Effect of Music-Based Intervention on Depression and Anhedonia. Front Psychol 2019, $10(1664$ 1078 (Print)):1038.

25. Grocke D, Bloch S, Castle D. The effect of group music therapy on quality of life for participants living with a severe and enduring mental illness. J Music Ther. 2009;46(2):90-104.

26. Raglio A, Attardo L, Gontero G, Rollino S, Groppo E, Granieri E. Effects of music and music therapy on mood in neurological patients. World journal of psychiatry. 2015;5(1):68-78.

27. Ekers D, Richards D, Gilbody S. A meta-analysis of randomized trials of behavioural treatment of depression. Psychological medicine. 2008;38(5):611-23. 
28. Young KS, Hasratian AM, Parsons CE, Zinbarg RE, Nusslock R, Bookheimer SY, Craske MG: Positive social feedback alters emotional ratings and reward valuation of neutral faces. Quarterly journal of experimental psychology (2006) 2019:1747021819890289.

29. Admon R, Pizzagalli DA. Dysfunctional Reward Processing in Depression. Curr Opin Psychol. 2015;4(Print):2352-250X. 114-118.

30. Robbins TW, Everitt BJ. Neurobehavioural mechanisms of reward and motivation. Curr Opin Neurobiol. 1996;6(2):228-36.

31. Saunders BT, Richard JM, Janak PH. Contemporary approaches to neural circuit manipulation and mapping: focus on reward and addiction. Philosophical transactions of the Royal Society of London Series B Biological sciences. 2015;370(1677):20140210.

32. Kabir ZD, Lee AS, Burgdorf CE, Fischer DK, Rajadhyaksha AM, Mok E, Rizzo B, Rice RC, Singh K, Ota KT, et al: Cacna1c in the Prefrontal Cortex Regulates Depression-Related Behaviors via REDD1. Neuropsychopharmacology 2017, 42(10):2032-2042.

33. Young CB, Chen T, Nusslock R, Keller J, Schatzberg AF, Menon V. Anhedonia and general distress show dissociable ventromedial prefrontal cortex connectivity in major depressive disorder. Transl Psychiatry. 2016;6:2158-3188. (Electronic)):810.

34. Der-Avakian A, Markou A. The neurobiology of anhedonia and other reward-related deficits. Trends Neurosci. 2012;35(1):68-77.

35. Pizzagalli DA. Depression, stress, and anhedonia: toward a synthesis and integrated model. Annu Rev Clin Psychol. 2014;10(Electronic):1548-5951. 393-423.

36. Treadway MT, Zald DH. Reconsidering anhedonia in depression: lessons from translational neuroscience. Neurosci Biobehav Rev. 2011;35(3):537-55.

37. Dellu F, Mayo W, Cherkaoui J, Le Moal M, Simon H. A two-trial memory task with automated recording: study in young and aged rats. Brain research. 1992;588(1):132-9.

38. Overstreet DH. Modeling depression in animal models. Methods in molecular biology. (Clifton $\mathrm{NJ}$ ). 2012;829:125-44.

39. Willner P, Towell A, Sampson D, Sophokleous S, Muscat R. Reduction of sucrose preference by chronic unpredictable mild stress, and its restoration by a tricyclic antidepressant. Psychopharmacology. 1987;93(3):358-64.

40. Ma K, Guo L, Xu A, Cui S, Wang JH. Molecular Mechanism for Stress-Induced Depression Assessed by Sequencing miRNA and mRNA in Medial Prefrontal Cortex. PloS one. 2016;11(7):e0159093.

41. Duman CH. Models of depression. Vitamins hormones. 2010;82:1-21.

42. Sun $X$, Song Z, Si Y, Wang JH. microRNA and mRNA profiles in ventral tegmental area relevant to stress-induced depression and resilience. Prog Neuro-psychopharmacol Biol Psychiatry. 2018;86:150-65.

43. Xu A, Cui S, Wang JH. Incoordination among Subcellular Compartments Is Associated with Depression-Like Behavior Induced by Chronic Mild Stress. The international journal of 
neuropsychopharmacology 2016, 19(5).

44. Ma K, Xu A, Cui S, Sun MR, Xue YC, Wang JH. Impaired GABA synthesis, uptake and release are associated with depression-like behaviors induced by chronic mild stress. Translational psychiatry. 2016;6(10):e910.

45. Qiao Y, Mao Y, Wang J, Chen R, Libing Z, Su YQ, Chen J, Zheng WQ. Analysis of liver and gill miRNAs of Larimichthys crocea against Cryptocryon irritans challenge. Fish Shellfish Immunol. 2016;59:48491.

46. He J, Chen Q, Wei Y, Jiang F, Yang M, Hao S, Guo X, Chen D, Kang L. MicroRNA-276 promotes egghatching synchrony by up-regulating brm in locusts. Proc Natl Acad Sci USA. 2016;113(3):584-9.

47. Si Y, Song Z, Sun $X$, Wang JH. microRNA and mRNA profiles in nucleus accumbens underlying depression versus resilience in response to chronic stress. American journal of medical genetics Part $B$, Neuropsychiatric genetics: the official publication of the International Society of Psychiatric Genetics 2018, 177(6):563-579.

48. Shen $M$, Song Z, Wang JH. microRNA and mRNA profiles in the amygdala are associated with stressinduced depression and resilience in juvenile mice. Psychopharmacology. 2019;236(7):2119-42.

49. Reid I, Forbes N, Stewart C, Matthews K. Chronic mild stress and depressive disorder: a useful new model? Psychopharmacology 1997, 134(4):365-367; discussion 371 - 367.

50 . Southwick SM, Charney DS. The science of resilience: implications for the prevention and treatment of depression. Science. 2012;338(6103):79-82.

51. Issler 0 , Chen A. Determining the role of microRNAs in psychiatric disorders. Nature reviews Neuroscience. 2015;16(4):201-12.

52. Bartel DP. MicroRNAs: target recognition and regulatory functions. Cell. 2009;136(2):215-33.

53. Luyten P, Fonagy P. The stress-reward-mentalizing model of depression: An integrative developmental cascade approach to child and adolescent depressive disorder based on the Research Domain Criteria (RDoC) approach. Clin Psychol Rev. 2018;64:87-98.

54. Li HX, Long QS, Chen AT, Li Q. [The influence of reward motivation on emotion regulation]. Sheng Li Xue Bao. 2019;71(4):562-74.

55. Otte C, Gold SM, Penninx BW, Pariante CM, Etkin A, Fava M, Mohr DC, Schatzberg AF. Major depressive disorder. Nature reviews Disease primers. 2016;2:16065.

56. van den Brink RHS, Schutter N, Hanssen DJC, Elzinga BM, Rabeling-Keus IM, Stek ML, Comijs HC, Penninx B, Oude Voshaar RC. Prognostic significance of social network, social support and Ioneliness for course of major depressive disorder in adulthood and old age. Epidemiology psychiatric sciences. 2018;27(3):266-77.

57. Santini ZI, Koyanagi A, Tyrovolas S, Mason C, Haro JM. The association between social relationships and depression: a systematic review. J Affect Disord. 2015;175:53-65.

\section{Figures}




\begin{tabular}{|c|c|}
\hline Control & No stress $\longrightarrow$ Control mice \\
\hline CUMS & Chronic unpredictable mild stress $\longrightarrow$ CUMS-MDD/CUMS-resilience mice \\
\hline CUMS-companion & Chronic unpredictable stress + companion $\longrightarrow$ Reward-MDD/Reward-resilience mice \\
\hline
\end{tabular}

4 week CUMS treatment

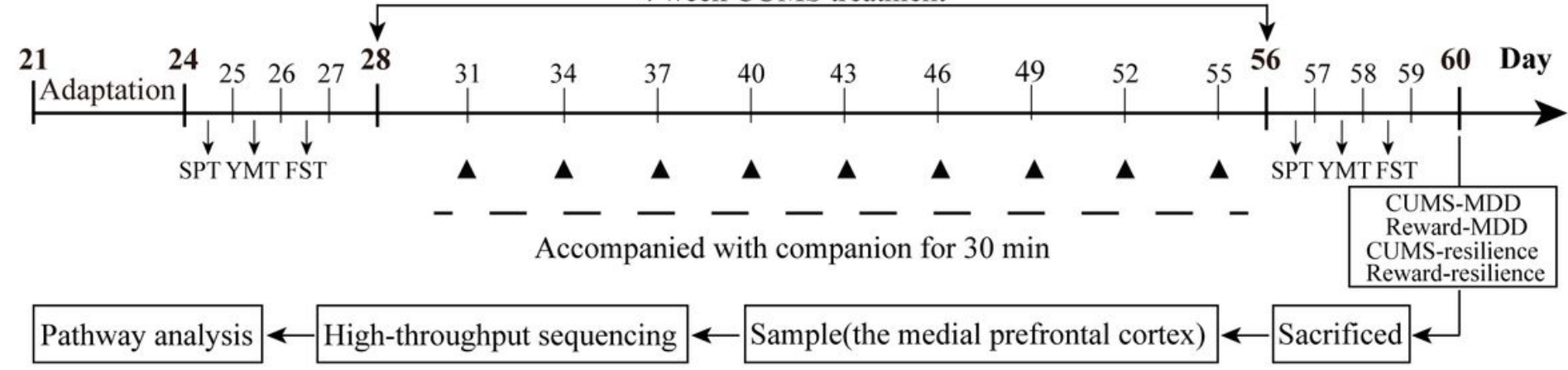

b

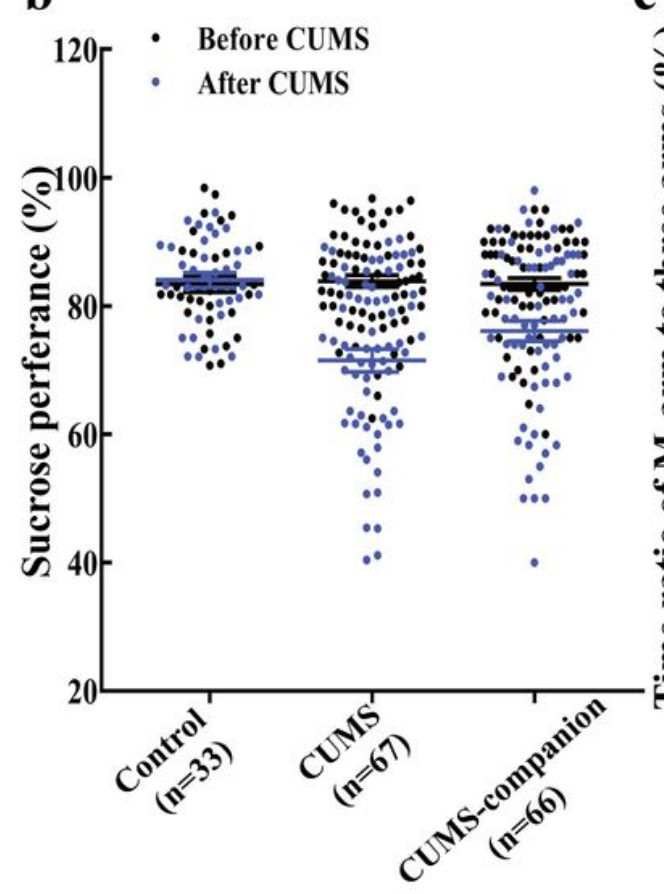

c

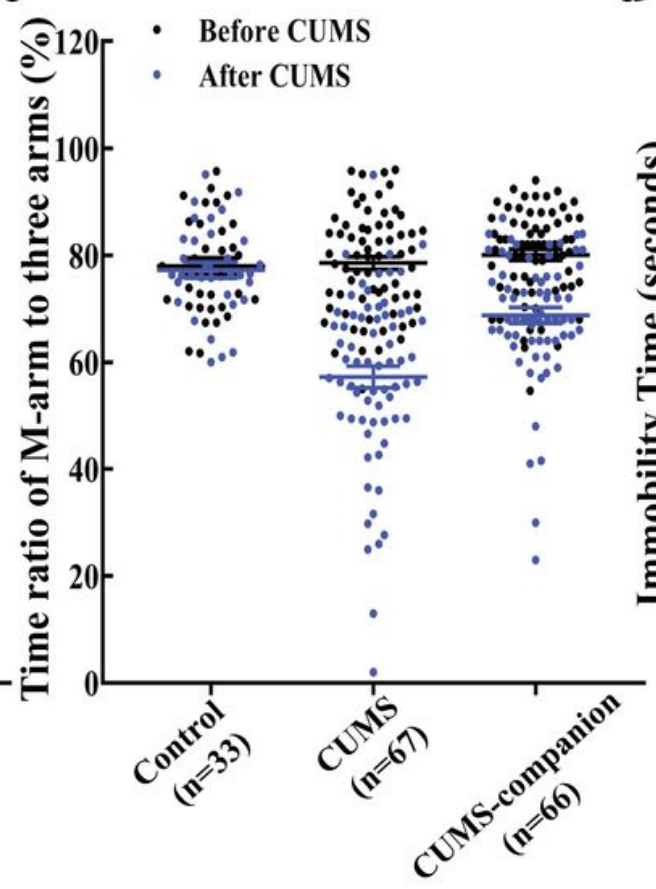

d

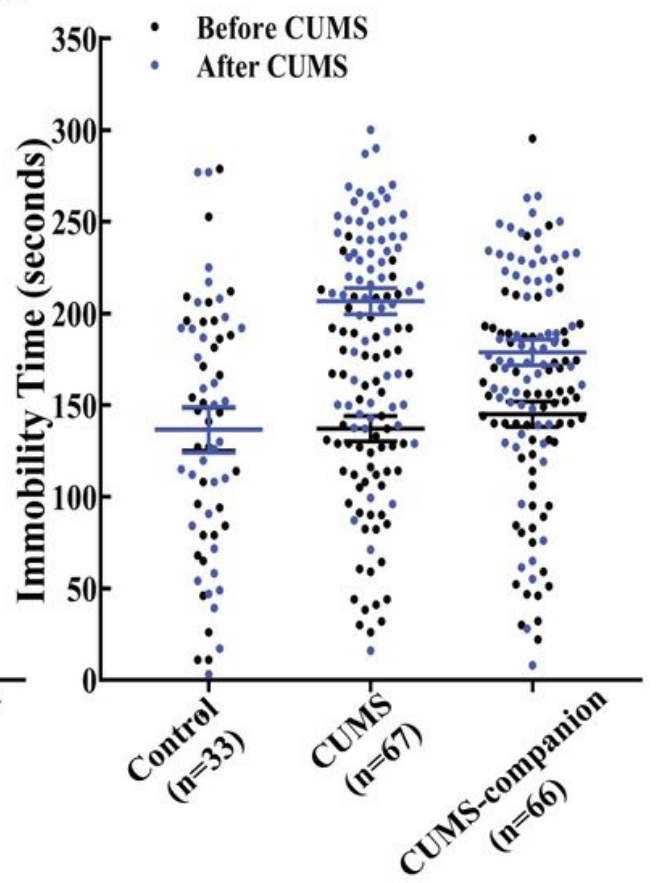

Figure 1

Accompanying with companion have ameliorated effort to CUMS-induced depression-like behaviors. a The CUMS and CUMS-companion procedures to produce CUMS-MDD, CUMS-resilience, Reward-MDD and Reward-resilience mice. These procedures including adaptation, behavioral tests, CUMS or CUMS with accompanying with companion treatments. b-d Sucrose preference test values (\%), ratios of stay time in M-arm to stay time in three arms by the YMT, immobile time of staying in the water cylinder by the FST in the mice from control group $(n=33)$, CUMS group $(n=67)$ and CUMS-companion group $(n=66)$ before (black) and after treatments (blue). Two-way ANOVA was used for the comparisons among control, CUMS and CUMS-companion groups. Specific analysis was shown in Table S1-S3. 
Metabolic pathways

Aldh1a2^ B3gnt5 $\downarrow \quad$ Pygm $\uparrow$

Aldh3b1^ Dpm3 $\uparrow$ Tyms $\uparrow$

Aldob $\downarrow \quad$ Folh1 $\downarrow \quad$ Uqcr11 $\uparrow$

Anpep $\uparrow \quad$ Polr2i $\uparrow \quad$ Pik3c2a

Aspa $\downarrow \quad$ Prodh $\downarrow \quad$ Polr2l $\uparrow$

Atp5k^ Ptgds $\uparrow$

B3galt4^ Ndufa13 $\uparrow$

PI3K-Akt signaling pathway

Collal $\uparrow \quad$ Myb $\downarrow \quad$ Sgk1 $\downarrow$

Col3al $\uparrow \quad N r 4 a 1 \uparrow$

I17r $\downarrow \quad$ Vtn $\uparrow$

Wnt signaling pathway

Bambi $\uparrow \quad$ Gm5415^

Fzd4 $\downarrow \quad$ Gm9839^

Hippo signaling pathway

Bmp6^ ${ }^{\wedge} \quad$ Fzd4 $\downarrow$

Bmp7^ Mob1b $\downarrow$

Huntington's disease

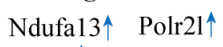

Polr $2 \mathrm{i}^{\uparrow} \quad$ Uqcr11 $\uparrow$

Cytokine-cytokine receptor interaction

Bmp7^ II7r $\downarrow$

Cor10^ Il1r1
Alcoholism

Hist1h2ap^ Crh^

Hist1h2ao^

Glutathione metabolism

Anpep $\uparrow$ Gstm $2 \uparrow$ Gstt3^

ECM-receptor interaction Collal $\uparrow$ Col3al^ $\operatorname{Vtn}^{\uparrow}$

MAPK signaling pathway Map3k6 $\downarrow$ Nr4al^ Illrl $\downarrow$

TGF-beta signaling pathway

Bambi $\uparrow \quad B m p 6 \uparrow$ Bmp7 $\uparrow$

Parkinson's disease

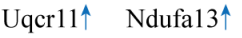

Alzheimer's disease

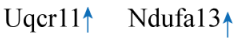

Histidine metabolism

Aldh3b1^ Aspa $\downarrow$

Long-term depression

Gucyla $2 \downarrow \mathrm{Crh}^{\uparrow}$

Circadian entrainment

Gucy $1 \mathrm{a} 2 \downarrow$ Per $2 \downarrow$
FoxO signaling pathway

Sgk1 $\quad$ Il7r $\downarrow$

Renin-angiotensin system

Anpep $\uparrow \quad$ Lnpep $\downarrow$

Thyroid hormone signaling pathway

Dio2 $\quad$ Slc2a1 $\downarrow$

Neuroactive ligand-receptor interaction

Hertr $2 \downarrow \quad$ Rxfp $2 \downarrow$

Alanine, aspartate and glutamate metabolism

Aspa $\quad$ Folh1 $\downarrow$

Circadian rhythm

Per2 $\downarrow$

Axon guidance

Sema $3 b \uparrow$

cGMP-PKG signaling pathway

Gucy1a2 $\downarrow$

Adrenergic signaling in cardiomyocytes

$\operatorname{Scn} 5 \mathrm{a} \uparrow$

Inflammatory mediator regulation of TRP channels I11r1 $\downarrow$

\section{Control vs CUMS-MDD}

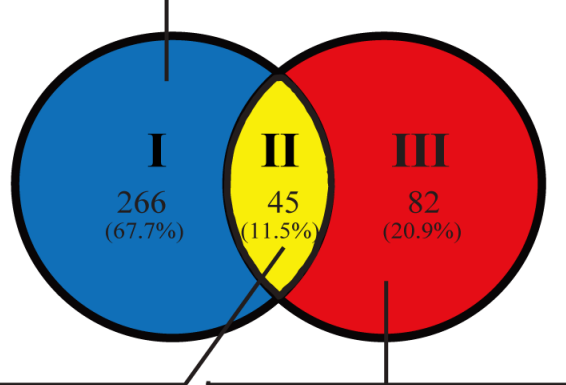

Control vs Reward-MDD

\begin{tabular}{|c|c|c|}
\hline \multicolumn{2}{|c|}{ Metabolic pathways } & Rap1 signaling pathway \\
\hline \multirow{2}{*}{$\begin{array}{l}\text { Etnppl } \downarrow \\
\text { Gpt } \downarrow \downarrow\end{array}$} & \multirow{2}{*}{$\begin{array}{l}\text { Ugt8a } \downarrow \\
\text { Xdh } \downarrow\end{array}$} & $\mathrm{Kdr} \uparrow \uparrow$ \\
\hline & & Hippo signaling pathway \\
\hline \multicolumn{2}{|c|}{ cAMP signaling pathway } & Crb1 $\downarrow$ \\
\hline Hhip $\downarrow$ & $\operatorname{Htr} 4 \downarrow \downarrow$ & $\begin{array}{l}\text { Calcium signaling pathway } \\
\text { Htr } 4 \downarrow \downarrow\end{array}$ \\
\hline \multicolumn{2}{|c|}{ ECM-receptor interaction } & \\
\hline $\mathrm{Cd} 44 \uparrow \uparrow$ & $\operatorname{Sdc} 4 \downarrow \downarrow$ & $\begin{array}{l}\text { TGF-beta signaling pathway } \\
\text { Smad6 } 6 \uparrow\end{array}$ \\
\hline \multicolumn{2}{|c|}{$\begin{array}{l}\text { Serotonergic synapse } \\
\text { Htr4 } \downarrow\end{array}$} & $\begin{array}{l}\text { PI3K-Akt signaling pathway } \\
\mathrm{Kdr} \uparrow \uparrow\end{array}$ \\
\hline \multicolumn{2}{|c|}{$\begin{array}{l}\text { Caffeine metabolism } \\
\text { Xdh } \downarrow\end{array}$} & $\begin{array}{l}\text { Chemokine signaling pathway } \\
\mathrm{Ccl} 28 \downarrow \downarrow\end{array}$ \\
\hline
\end{tabular}

Cytokine-cytokine receptor interaction Ccl28 $\downarrow \quad$ I112rb1 $\downarrow$

Neuroactive ligand-receptor interaction $\mathrm{Htr} 4 \downarrow$

Alanine, aspartate and glutamate metabolism Gpt $\downarrow$

\begin{tabular}{|c|c|c|c|c|}
\hline \multicolumn{2}{|c|}{ Metabolic pathways } & \multirow{2}{*}{\multicolumn{2}{|c|}{$\begin{array}{l}\text { Alcoholism } \\
\text { Ddc } \downarrow\end{array}$}} & \multirow{2}{*}{$\begin{array}{l}\text { Dopaminergic synapse } \\
\text { Ddc } \downarrow\end{array}$} \\
\hline \multirow{2}{*}{$\begin{array}{l}\text { Acer } 2 \downarrow \\
\operatorname{Dmgdh} \uparrow\end{array}$} & \multirow{2}{*}{$\begin{array}{l}\text { Ak } 7 \downarrow \\
\text { Ddc } \downarrow\end{array}$} & & & \\
\hline & & \multicolumn{2}{|c|}{ Renin secretion } & Wnt signaling pathway \\
\hline Aldh1a $3 \downarrow$ & Chdh $\downarrow$ & \multicolumn{2}{|c|}{ Adrb $2 \downarrow$} & Wnt $3 \downarrow$ \\
\hline \multicolumn{2}{|c|}{ Tyrosine metabolism } & \multicolumn{2}{|c|}{ Cocaine addiction } & Tryptophan metabolism \\
\hline Aldh $1 \mathrm{a} 3 \uparrow$ & $\mathrm{Ddc} \downarrow$ & \multicolumn{2}{|c|}{$\mathrm{Ddc} \downarrow$} & Ddc $\downarrow$ \\
\hline \multicolumn{2}{|c|}{ MAPK signaling pathway } & \multirow{2}{*}{\multicolumn{2}{|c|}{$\begin{array}{l}\text { Alzheimer's disease } \\
\text { Fas } \downarrow\end{array}$}} & Ampheta \\
\hline $\operatorname{Relb} \uparrow$ & Fas $\downarrow$ & & & Ddc $\downarrow$ \\
\hline \multicolumn{2}{|c|}{ ECM-receptor interaction } & \multirow{2}{*}{\multicolumn{2}{|c|}{$\begin{array}{l}\text { Huntington's disease } \\
\text { Dnah6 } \downarrow\end{array}$}} & Hippo signaling path \\
\hline Itgal $\downarrow$ & Thbs $4 \downarrow$ & & & Wnt $3 \downarrow$ \\
\hline \multicolumn{2}{|c|}{ Phenylalanine metabolism } & \multirow{2}{*}{\multicolumn{2}{|c|}{$\begin{array}{l}\text { Serotonergic synapse } \\
\text { Ddc } \downarrow\end{array}$}} & cAMP sig \\
\hline Aldh $1 \mathrm{a} 3 \uparrow$ & Ddc $\downarrow$ & & & Adrb2 $\downarrow$ \\
\hline \multicolumn{2}{|c|}{ PI3K-Akt signaling pathway } & \multicolumn{2}{|c|}{ Histidine metabolism } & Calcium signaling pathwa \\
\hline Itgal $\downarrow$ & Thbs $4 \downarrow$ & Aldhla3 $\uparrow$ & & Adrb $2 \downarrow$ \\
\hline \multicolumn{3}{|c|}{$\begin{array}{l}\text { cGMP-PKG signaling pathway } \\
\text { Adrb } 2 \downarrow\end{array}$} & \multicolumn{2}{|c|}{$\begin{array}{l}\text { Cytokine-cytokine receptor interaction } \\
\text { Fas } \downarrow\end{array}$} \\
\hline \multicolumn{3}{|c|}{$\begin{array}{l}\text { Regulation of actin cytoskeleton } \\
\text { Itgal } \downarrow\end{array}$} & \multicolumn{2}{|c|}{$\begin{array}{l}\text { Neuroactive ligand-receptor interaction } \\
\text { Adrb } 2 \downarrow\end{array}$} \\
\hline
\end{tabular}

Figure 2

Signaling pathways identified by KEGG function analysis based on the differently expression genes (DEGs) between control versus CUMS-MDD and control versus Reward-MDD. Numbers and Venn diagram display of differently expression genes (DEGs) in control versus CUMS-MDD and control versus RewardMDD. $\otimes$ (blue) shows the certain genes specifically involved in CUMS-MDD mice, $\otimes$ (yellow) indicates the common genes changed in both CUMS-MDD and Reward-MDD mice, $\otimes$ (red) shows the certain genes 
specifically involved in Reward-MDD mice. $\uparrow$ (blue) indicates up-regulation in CUMS-MDD mice, whereas $\downarrow$ (blue) represents down-regulation. $\uparrow$ (red) indicates up-regulation in Reward-MDD mice, whereas $\downarrow$ (red) represents down-regulation.

cAMP signaling pathway

Glp1r $\uparrow$ Nfatc1 $\downarrow$ Sstr5 $\uparrow$

Metabolic pathways

Lipg $\uparrow$

Wnt signaling pathway

Nfatcl $\downarrow$

ECM-receptor interaction Itgb4 $\downarrow$
Tight junction

Crb3 $\downarrow$

MAPK signaling pathway

Nfatc1 $\downarrow$

PI3K-Akt signaling pathway Itgb4 $\downarrow$

Regulation of actin cytoskeleton Itgb4 $\downarrow$
cGMP-PKG signaling pathway

Nfatc1 $\downarrow$

Neuroactive ligand-receptor interaction

Glp1r $\uparrow \quad$ Ltb4r1 $\uparrow \quad$ Sstr5 $\uparrow$

Cytokine-cytokine receptor interaction Cxcl10 $\downarrow$

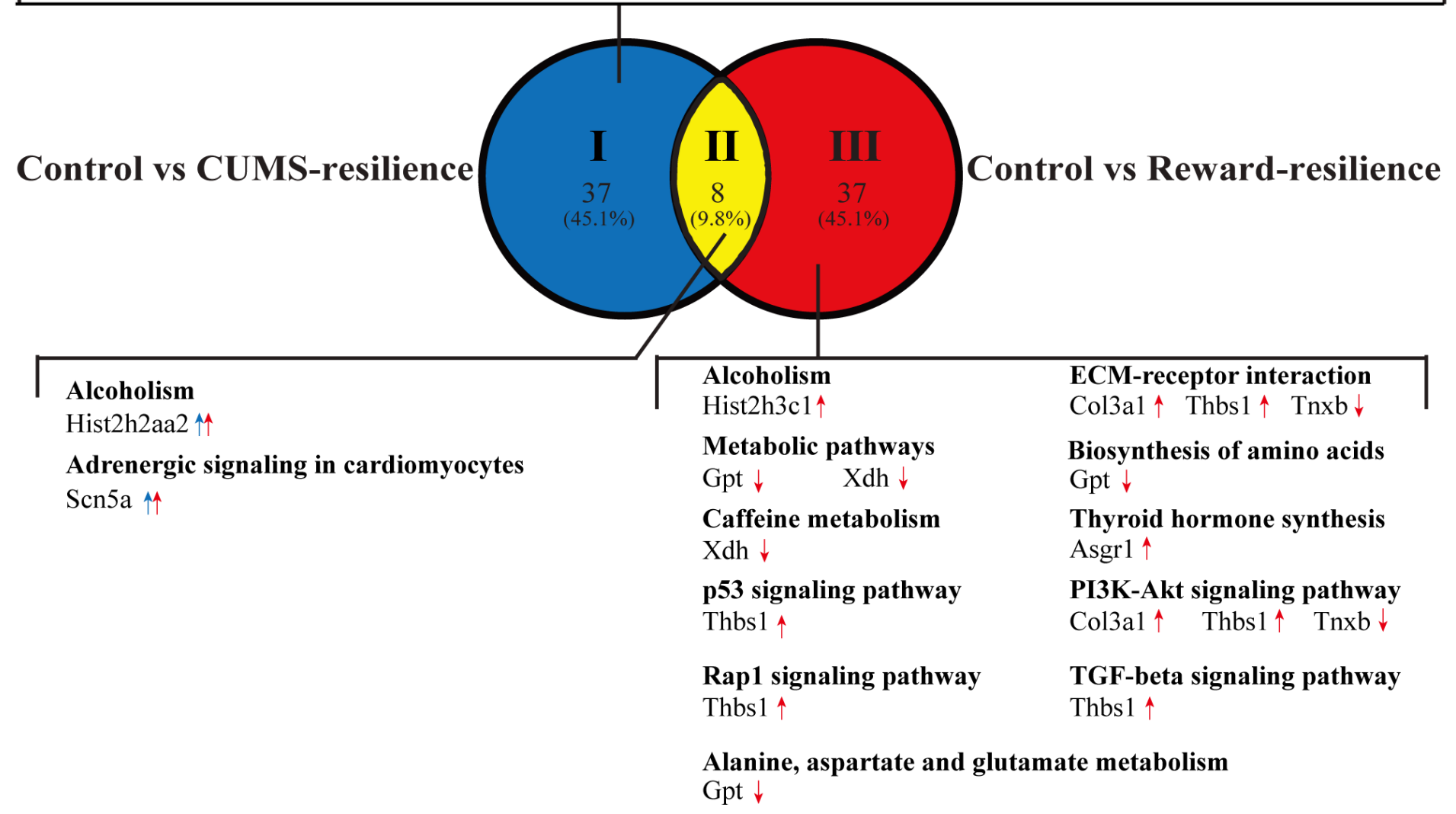

Figure 3

Signaling pathways identified by KEGG function analysis based on the differently expression genes (DEGs) between control versus CUMS-resilience and control versus Reward-resilience. Numbers and Venn diagram display of differently expression genes (DEGs) in control versus CUMS-resilience and control

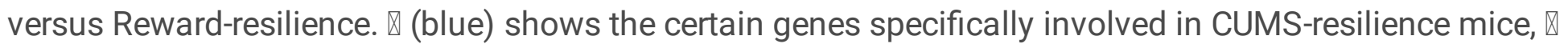

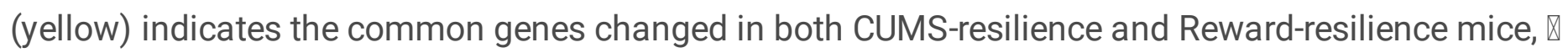
(red) shows the certain genes specifically involved in Reward-resilience mice. $\uparrow$ (blue) indicates upregulation in CUMS-resilience mice, whereas $\downarrow$ (blue) represents down-regulation. $\uparrow$ (red) indicates upregulation in Reward-resilience mice, whereas $\downarrow$ (red) represents down-regulation. 


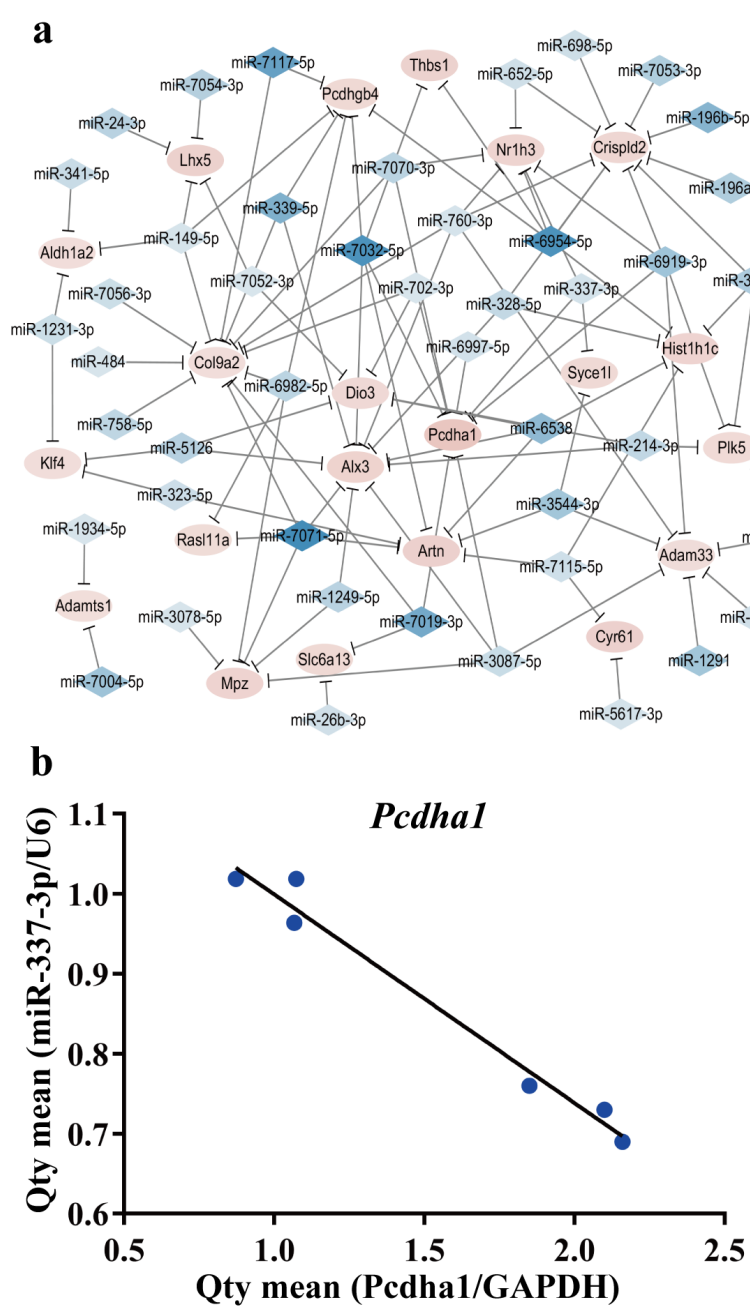

b

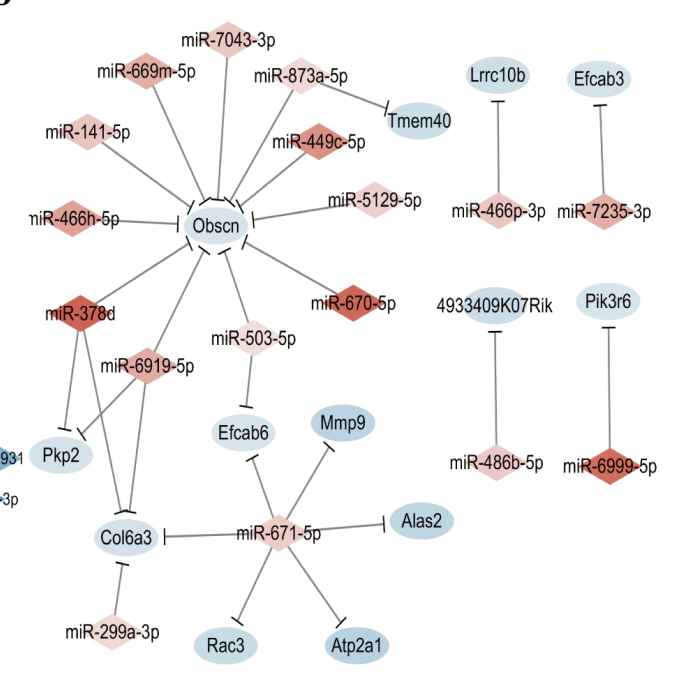

d
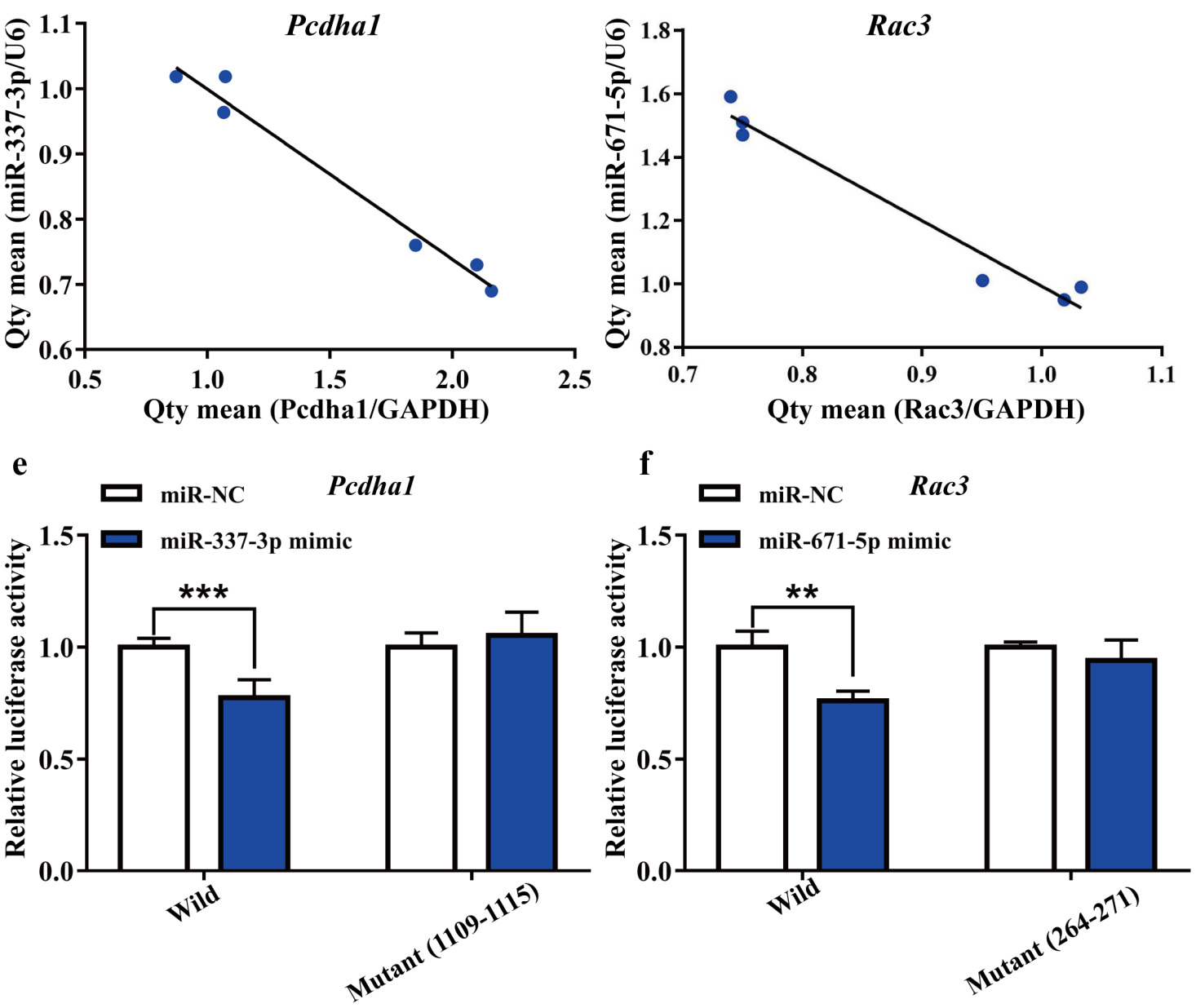

Figure 4

Differently expression miRNAs targeted differently expression mRNAs in CUMS-resilience versus Rewardresilience mice. a-b MicroRNA-mRNA network in CUMS-resilience versus Reward-resilience mice were constructed between the 63 miRNAs and 34 overlapped mRNAs with using transcriptome expression data and predicted target genes from RNAhybrid, Targetscan, and miRanda databases. Red symbols present the elevated expression of miRNAs or mRNAs. Blue symbols present the down regulated miRNAs or 
mRNAs. c-d miRNAs and its targeted mRNAs were validated by qRT-PCR assay, and the correlation between them were analyzed. $c$ The correlation between Pcdha1 and miR-337-3p $(r=-0.988 ; p=$ $0.0002)$. $d$ The correlation between Rac3 and miR-671-5p ( $r=-0.981 ; p=0.0005)$. e-f miRNAs and its targeted mRNAs were validated by Luciferase reporter assay. e Luciferase reporter assay performed by the co-transfection of luciferase reporter containing wild or mutant of Pcdha1 mRNA with miR-337-3p mimic or negative control (NC) into HEK293T cells. $f$ Luciferase reporter assay performed by the cotransfection of luciferase reporter containing wild or mutant of Rac3 mRNA with miR-671-5p mimic or negative control (NC) into HEK293T cells. Data are the mean \pm SEM.

\section{Supplementary Files}

This is a list of supplementary files associated with this preprint. Click to download.

- Additionalfiles2TableS7.xlsx

- Additionalfiles2TableS5.xIsx

- Additionalfiles1.pdf

- Additionalfiles2TableS8.xIsx

- ChecklistS1.docx

- Additionalfiles2TableS6.xIsx 